\title{
The quark core of protoneutron stars in the phase diagram of quark matter
}

\author{
F. Sandin ${ }^{1, *}$ and D. Blaschke $2,3,4$, 丹 \\ 1 Department of Physics, Luleå University of Technology, SE-97187 Luleå, Sweden \\ ${ }^{2}$ Instytut Fizyki Teoretycznej, Uniwersytet Wrockawski, PL-50-204 Wrockaw, Poland \\ ${ }^{3}$ Bogoliubov Laboratory for Theoretical Physics, JINR Dubna, RU-141980 Dubna, Russia \\ ${ }^{4}$ Institut für Physik, Universität Rostock, D-18051 Rostock, Germany
}

\begin{abstract}
We study the effect of neutrino trapping in new-born quark stars within a three-flavor NambuJona-Lasinio (NJL) model with self-consistently calculated quark masses. The phase diagrams and equations of state for charge neutral quark matter in $\beta$-equilibrium are presented, with and without trapped neutrinos. The compact star sequences for different neutrino untrapping scenarios are investigated and the energy release due to neutrino untrapping is found to be of the order of $10^{53} \mathrm{erg}$. We find that hot quark stars characterized, e.g., by an entropy per baryon of $1-2$ and a lepton fraction of 0.4 , as models for the cores of newborn protoneutron stars, are in the two-flavor color superconducting (2SC) state. High temperatures and/or neutrino chemical potentials disfavor configurations with a color-flavor-locked (CFL) phase. Stable quark star solutions with CFL cores exist only at low temperatures and neutrino chemical potentials.

PACS numbers: 12.38.Aw, 12.39.-x, 24.85.+p, 26.60.+c, 97.60.-s
\end{abstract}

\section{INTRODUCTION}

The engine of explosive phenomena in astrophysics, such as gamma-ray bursts (GRBs) and type-II supernovæ is presently not fully understood. The generation and propagation of neutrino fluxes, as well as the neutrino interactions in the hot and dense nuclear matter envelope play key roles in models of the evolution of core-collapse supernovæ [1]. Detailed hydrodynamic simulations of the gravitational collapse of a massive star show that the possibility to obtain a successful explosion depends to a large extent on the properties of the protoneutron star (PNS) that forms in the compressed core. About $99 \%$ of the gravitational binding energy is released by neutrino emission [2, 3, 4]. The supernova collapse and prompt neutrino production proceeds within milliseconds and the shock-compressed matter is heated up to about $30-50 \mathrm{MeV}$. At such high temperature the neutrino mean free path is much shorter than the radius of a PNS, $R \sim 10 \mathrm{~km}$, and neutrinos diffuse on a time scale of $\sim 10$ seconds.

During this 'neutrino trapping' regime the number of neutrinos is quasi conserved and the neutrino chemical potential, $\mu_{\nu}$, is of the order $200 \mathrm{MeV}[3$, 4]. This state lasts until the temperature is low enough for the neutrino mean free path to become comparable to $R$. The behavior of the neutrino mean free path, which determines the timescale for the untrapping transition and the onset of the 'second shock' of the supernova, depends in a sensitive way on the microphysics of the hot and dense PNS interior. In particular, if superfluid and superconducting phases of hadronic and/or quark matter are created, the transport properties are affected and the consequences

\footnotetext{
*Electronic address: fredrik.sandin@ltu.se

${ }^{\dagger}$ Electronic address: blaschke@ift.uni.wroc.pl
}

for simulations of core-collapse supernovæ could be important.

In a scenario where color superconducting quark matter is the preferred state of matter at high density there are several new aspects to consider, for example:

1. The kinetics of the phase transition, which eventually proceeds with the release of latent heat and requires a nucleation time scale.

2. The possibility that color superconducting phases have large pairing gaps $(\sim 100 \mathrm{MeV})$, which affect the transport properties in the PNS core.

3 . The possibility that color superconducting phases have a high critical temperature ( $\propto$ the gaps) and therefore affect the formation and evolution of PNS.

For recent reviews on dense color superconducting quark matter, see [5, 6, 7, 8, 9, 10, 11, 12] and references therein.

The energy released due to a phase transition to deconfined quark matter in a PNS core can reach the order of 100 bethe $\left(=10^{53} \mathrm{erg}\right)[13,14,15$, which is the correct order of magnitude for the energy of GRBs. Moreover, the nucleation timescale for a quark matter phase transition could explain the time delay statistics of GRB subpulse structure [16, 17]. It has also been emphasized that in the presence of a strong magnetic field, neutrinos propagating in hot superconducting quark matter can become collimated (beaming) and asymmetric, thus explaining a resulting kick velocity for the PNS [18].

In the present work we consider a microscopic, albeit schematic chiral quark model of NJL type, where the quark masses and pairing gaps are calculated selfconsistently at the mean-field level, see $[19,20,21,22$, 23]. We describe the phase structure of color superconducting three-flavor quark matter for two different strengths of the phenomenological diquark pairing interaction, in systems with and without trapped neutrinos. 
The corresponding quark star solutions are considered as models for PNS cores and their properties are described. In particular, we are interested in the answers to the following questions:

1. What influence has the neutrino chemical potential on the phase diagram of quark matter?

2. In which regions of the quark matter phase diagram can stable quark stars be found?

3. Can both two-flavor superconductivity (2SC) and three-flavor color-flavor-locking (CFL) phases be realized under PNS conditions?

4. How much energy can be released in the cooling and untrapping evolution for these models of a PNS core?

The effect of neutrino trapping on the phase structure of color superconducting three-flavor quark matter has been investigated before in Refs. [24, 25], for homogeneous phases, and in Ref. [26] for inhomogeneous phases of the LOFF type [27]. In this paper we focus on the stability of quark stars for different phase structures and estimates of the energy release in the cooling and untrapping transitions.

The paper is organized as follows. In Section II we define the NJL-type model that we use to describe color superconducting quark matter with trapped neutrinos. Section III gives a summary of all results, which are discussed in detail in subsections according to the main questions posed in the Introduction. The Conclusions summarize our main findings.

\section{MODEL OF HOT QUARK MATTER WITH TRAPPED NEUTRINOS}

Due to the high density in compact stars, strange quarks could exist in their interior. We therefore consider a grand canonical ensemble with up, down, and strange quark degrees of freedom. Because strange quarks have a relatively high mass, they should arise only at high density. Therefore, at not too high densities, where matter is in a two-flavor state, there should be an excess of down quarks for matter to be charge neutral. Consequently, the difference between the chemical potentials of the up and down quarks can be sufficiently large for muons to be created by weak interactions, $d \leftrightarrow u+\mu^{-}+\bar{\nu}_{\mu}$. It is therefore necessary to include both electron and muon lepton degrees of freedom in the model. The $\tau$ lepton is, however, not included, because it is too massive to play a significant role in compact stars. We neglect the influence of neutrino oscillations and therefore omit the $\tau$ neutrino as well.

The thermodynamics of the quark matter phase is described with an NJL-type model. The path-integral representation of the quark partition function is [20, 21, 22]

$$
\begin{aligned}
Z(T, \hat{\mu}) & =\int \mathcal{D} \bar{q} \mathcal{D} q e^{\int d^{4} x\left(\mathcal{L}_{f}+\mathcal{L}_{\bar{q} q}+\mathcal{L}_{q q}\right)} \\
\mathcal{L}_{f} & =\bar{q}\left(i \not \partial-\hat{m}+\hat{\mu} \gamma^{0}\right) q \\
\mathcal{L}_{\bar{q} q} & =G_{S} \sum_{a=0}^{8}\left(\bar{q} \tau_{a} q\right)^{2} \\
\mathcal{L}_{q q} & =G_{D} \sum_{A=2,5,7}\left(\bar{q} i \gamma_{5} \tau_{A} \lambda_{A} C \bar{q}^{T}\right)\left(q^{T} i C \gamma_{5} \tau_{A} \lambda_{A} q\right)
\end{aligned}
$$

where $\hat{\mu}$ and $\hat{m}$ are the diagonal chemical potential and current quark mass matrices in color and flavor space. For $a=0, \tau_{0}=(2 / 3)^{1 / 2} \mathbf{1}_{f}$, otherwise $\tau_{a}$ and $\lambda_{a}$ are Gell-Mann matrices acting in flavor and color space, respectively. $C=i \gamma^{2} \gamma^{0}$ is the charge conjugation operator and $\bar{q}=q^{\dagger} \gamma^{0}$. The coupling constants, $G_{S}$ and $G_{D}$, determine the coupling strengths in the $\bar{q} q$ and $q q$ channels, which represent current-current interactions in the color singlet scalar meson channel and the scalar color antitriplet diquark channel. We follow the argument in [21] concerning the $\mathrm{U}_{A}(1)$ symmetry breaking in the pseudoscalar isoscalar meson sector, which essentially is that the symmetry breaking is dominated by quantum fluctuations, and we therefore omit the 't Hooft determinant interaction. In the following we use the relative coupling strength

$$
\eta=G_{D} / G_{S}
$$

to parametrize the coupling in the diquark channel. In the choice of the four-fermion interaction channels we have omitted the pseudoscalar $\bar{q} q$ terms, which should be present in a chirally symmetric theory. These terms do not contribute to the thermodynamic properties of the deconfined quark matter phase at the mean-field (Hartree) level [23], to which we restrict the discussion in the present paper.

After bosonization using Hubbard-Stratonovich transformations, we obtain an exact transformation of the original partition function (11). The transformed expression constitutes the starting point for approximations, defined as truncations of the Taylor expanded action functional to different orders in the collective boson fields. In the following, we use the mean-field (MF) approximation. This means that the bosonic functional integrals are omitted and the collective fields are fixed at the extremum of the action. The corresponding mean-field thermodynamic potential, from which all thermodynamic quantities can be derived, is given by

$$
\begin{aligned}
\Omega_{\mathrm{MF}} & \left(T, \hat{\mu}, \mu_{L_{e}}, \mu_{L_{\mu}}\right) \\
= & -\frac{1}{\beta V} \ln Z_{\mathrm{MF}}(T, \hat{\mu})+\Omega_{l}\left(T, \mu_{L_{e}}, \mu_{L_{\mu}}\right) \\
& =\sum_{i=u, d, s} \frac{\left(M_{i}-m_{i}\right)^{2}}{8 G_{S}}+\frac{\Delta_{u d}^{2}+\Delta_{u s}^{2}+\Delta_{d s}^{2}}{4 \eta G_{S}} \\
& -\int \frac{d^{3} p}{(2 \pi)^{3}} \sum_{a=1}^{18}\left[E_{a}(p)+2 T \ln \left(1+e^{-E_{a}(p) / T}\right)\right]
\end{aligned}
$$




$$
+\Omega_{l}\left(T, \mu_{L_{e}}, \mu_{L_{\mu}}\right)-\Omega_{0} .
$$

Here, $M_{i}=m_{i}+\phi_{i}$ are the renormalized quark masses, $m_{i}$ are the current quark masses, $E_{a}(p)$ are the eighteen independent quark quasiparticle dispersion relations, and $\phi_{i}\left(\Delta_{i j}\right)$ are chiral (diquark) gaps, see [21] for details. The gaps, $\phi_{i}$ and $\Delta_{i j}$, emerge from the auxiliary boson fields introduced by the Hubbard-Stratonovich transformations and represent collective modes generated by $\bar{q} q$ and $q q$, respectively. $\Omega_{l}\left(T, \mu_{L_{e}}, \mu_{L_{\mu}}\right)$ is the thermodynamic potential for an ideal gas of neutrinos, electrons $\left(m_{e} \simeq 0.511 \mathrm{MeV}\right)$, and muons $\left(m_{\mu} \simeq 105.66 \mathrm{MeV}\right)$

$$
\begin{array}{r}
\Omega_{l}\left(T, \mu_{L_{e}}, \mu_{L_{\mu}}\right)=-\sum_{l=e, \mu}\left[\frac{\mu_{\nu_{l}}^{4}}{24 \pi^{2}}+\frac{\mu_{\nu_{l}}^{2} T^{2}}{12}+\frac{7 \pi^{2} T^{4}}{360}\right. \\
\left.+\frac{T}{\pi^{2}} \sum_{ \pm} \int_{0}^{\infty} \mathrm{d} k k^{2} \ln \left(1+e^{-\frac{E_{l} \pm \mu_{l}}{T}}\right)\right]
\end{array}
$$

where $E_{l}=\sqrt{p^{2}+m_{l}^{2}} . \quad \Omega_{0}$ is a divergent term that is subtracted in order to get zero pressure and energy density in vacuum (i.e., at $T=\mu=0$ )

$$
\Omega_{0}=\sum_{i=u, d, s} \frac{\left(\phi_{i}^{0}\right)^{2}}{8 G_{S}}-6 \int \frac{d^{3} p}{(2 \pi)^{3}} \sqrt{p^{2}+\left(m_{i}+\phi_{i}^{0}\right)^{2}} .
$$

The model has six conserved charge densities and associated chemical potentials. The $U(3)_{f} \times S U(3)_{c} \times U(1)_{Q}$ symmetry of the quarks is broken in the presence of diquarks. Therefore, there are only four mutually commuting conserved charge densities, e.g., the quark number density

$$
n=\left\langle q^{\dagger} q\right\rangle=-\frac{\partial \Omega_{\mathrm{MF}}}{\partial \mu}
$$

two color charge densities

$$
\begin{aligned}
& n_{3}=\left\langle q^{\dagger} \lambda_{3} q\right\rangle=-\frac{\partial \Omega_{\mathrm{MF}}}{\partial \mu_{3}}, \\
& n_{8}=\left\langle q^{\dagger} \lambda_{8} q\right\rangle=-\frac{\partial \Omega_{\mathrm{MF}}}{\partial \mu_{8}},
\end{aligned}
$$

and the electric charge density

$$
n_{Q}=\left\langle q^{\dagger} Q q\right\rangle-n_{e}-n_{\mu}=-\frac{\partial \Omega_{\mathrm{MF}}}{\partial \mu_{Q}} .
$$

Here, $Q=\operatorname{diag}_{f}\left(\frac{2}{3},-\frac{1}{3},-\frac{1}{3}\right)$ and $n_{e}\left(n_{\mu}\right)$ is the number density of electrons (muons). Consequently, the quark chemical potential matrix, $\hat{\mu}$, is

$$
\hat{\mu}=\mu+Q \mu_{Q}+\lambda_{3} \mu_{3}+\lambda_{8} \mu_{8},
$$

where $\mu$ is the quark number chemical potential, $\mu_{Q}$ the (positive) electric charge chemical potential, and $\mu_{3}$ and $\mu_{8}$ are color charge chemical potentials. Note the discussion of color neutrality in Ref. [28].
The quark number density is related to the baryon number density by

$$
n_{B}=n / 3 \text {. }
$$

The remaining two charges are the number densities of the lepton families

$$
\begin{aligned}
& n_{L_{e}}=n_{e}+n_{\nu_{e}}=-\frac{\partial \Omega_{\mathrm{MF}}}{\partial \mu_{L_{e}}}, \\
& n_{L_{\mu}}=n_{\mu}+n_{\nu_{\mu}}=-\frac{\partial \Omega_{\mathrm{MF}}}{\partial \mu_{L_{\mu}}},
\end{aligned}
$$

which are conserved when the neutrinos are trapped in the system and oscillations are neglected. Electrons and muons have both electric charge and lepton number, while neutrinos have lepton number only. Therefore

$$
\begin{aligned}
\mu_{e} & =\mu_{L_{e}}-\mu_{Q}, \\
\mu_{\mu} & =\mu_{L_{\mu}}-\mu_{Q}, \\
\mu_{\nu_{e}} & =\mu_{L_{e}}, \\
\mu_{\nu_{\mu}} & =\mu_{L_{\mu}} .
\end{aligned}
$$

The lepton fractions, which represent the relative number of leptons and baryons, are defined as

$$
\begin{aligned}
Y_{L_{e}} & \equiv \frac{n_{L_{e}}}{n_{B}}, \\
Y_{L_{\mu}} & \equiv \frac{n_{L_{\mu}}}{n_{B}} .
\end{aligned}
$$

Bulk matter in compact stars should be charge neutral. In NJL models there are no gauge fields that neutralize the color charge dynamically, because the gluons are replaced by effective point-like $\bar{q} q$ and $q q$ interactions. Color neutrality must therefore be enforced by solving for the charge chemical potentials, $\mu_{3}$ and $\mu_{8}$, such that the corresponding charge densities, $n_{a}=\left\langle q^{\dagger} \lambda_{a} q\right\rangle$, are zero. In addition, matter in compact stars should be electrically neutral and in $\beta$-equilibrium with respect to weak interactions. The chemical potentials, $\mu_{Q}, \mu_{3}$, and $\mu_{8}$ are therefore determined such that the charge densities (7)-(8) vanish

$$
\frac{\partial \Omega_{\mathrm{MF}}}{\partial \mu_{Q}}=\frac{\partial \Omega_{\mathrm{MF}}}{\partial \mu_{3}}=\frac{\partial \Omega_{\mathrm{MF}}}{\partial \mu_{8}}=0 .
$$

Observe that the definition of the quark chemical potential (9) implies that matter is in $\beta$-equilibrium. This holds true also when neutrinos are trapped and $\mu_{L_{l}}>0$, because, e.g., $\mu_{e}+\mu_{\bar{\nu}_{e}}=\left(\mu_{L_{e}}-\mu_{Q}\right)+\left(-\mu_{L_{e}}\right)=-\mu_{Q}$. The gaps, $\phi_{i}$ and $\Delta_{i j}$, are order parameters that are determined by minimization of the mean-field thermodynamic potential (3)

$$
\begin{gathered}
\frac{\partial \Omega_{\mathrm{MF}}}{\partial \phi_{u}}=\frac{\partial \Omega_{\mathrm{MF}}}{\partial \phi_{d}}=\frac{\partial \Omega_{\mathrm{MF}}}{\partial \phi_{s}}=0, \\
\frac{\partial \Omega_{\mathrm{MF}}}{\partial \Delta_{u d}}=\frac{\partial \Omega_{\mathrm{MF}}}{\partial \Delta_{u s}}=\frac{\partial \Omega_{\mathrm{MF}}}{\partial \Delta_{d s}}=0 .
\end{gathered}
$$

Local minima of the thermodynamic potential define competing phases and the global minimum is the physical solution. 
At the charge neutral global minimum of the thermodynamic potential the pressure, entropy density, number densities, and energy density are

$$
\begin{aligned}
P\left(T, \mu, \mu_{L_{e}}, \mu_{L_{\mu}}\right) & =-\Omega_{\mathrm{MF}}, \\
s\left(T, \mu, \mu_{L_{e}}, \mu_{L_{\mu}}\right) & =-\frac{\partial \Omega_{\mathrm{MF}}}{\partial T}, \\
n_{a}\left(T, \mu, \mu_{L_{e}}, \mu_{L_{\mu}}\right) & =-\frac{\partial \Omega_{\mathrm{MF}}}{\partial \mu_{a}}, \\
\epsilon\left(T, \mu, \mu_{L_{e}}, \mu_{L_{\mu}}\right) & =-P+T s+\sum_{a=B, L_{e}, L_{\mu}} \mu_{a} n_{a} .
\end{aligned}
$$

The sum in the expression for the energy density should account for all conserved charges in the system. However, because we require that $n_{Q}=n_{3}=n_{8}=0$, only three terms are included. Once the current quark masses, coupling constants, and momentum regularization method has been fixed, equations (2)-(5), (9), and (14)-(16) define a self-consistent set of equations for the quark matter model.

\section{A. Numerical methods}

The quark quasiparticle dispersion relations, $E_{a}(p)$, in (3) are eigenvalues of Hermitian matrices, see [21] for details. LAPACK is used to calculate the eigenvalues and the corresponding eigenvectors of these matrices. For given values of all parameters, the mean-field thermodynamic potential (3) is calculated with a Gaussian integration quadrature. Observe that the integrands depend only on the magnitude of the three-momentum, so $d^{3} p \rightarrow 4 \pi p^{2} d p$.

The derivatives of the thermodynamic potential are explicitly calculated using the eigenvectors of the Hermitian matrices. The derivatives are of the form

$$
\frac{\partial \Omega_{\mathrm{MF}}}{\partial x}=F_{1}(x)-\int \frac{d^{3} p}{(2 \pi)^{3}} \sum_{a=1}^{18} F_{2}\left(\frac{\partial E_{a}(p)}{\partial x}\right),
$$

for some simple functions $F_{1}$ and $F_{2}$ that depend on the choice of the variable $x$. The numerical problem therefore is to calculate the derivatives of the quasiparticle dispersion relations, $E_{a}(p)$, i.e., the derivatives of the eigenvalues of the Hermitian matrices. It is easy to show that

$$
\frac{\partial E_{a}}{\partial x}=\frac{X_{a}^{\dagger} \frac{\partial H}{\partial x} X_{a}}{X_{a}^{\dagger} X_{a}}
$$

where $X_{a}$ is the eigenvector associated to an eigenvalue $E_{a}$ of a Hermitian matrix $H$. The derivatives of the Hermitian matrices are sparse and all derivatives of the thermodynamic potential are therefore obtained practically at the cost of computing the eigenvectors. The integrands of all derivatives and the thermodynamic potential are calculated in parallel and the momentum integrals are evaluated simultaneously to reduce computational redundancy. The gap and charge neutrality equations (14)-(15) are solved with a modified Newton method in multidimensions, i.e., essentially a steepest descent method, for different starting points in parameter space. The solution with the highest pressure is used. The results thereby obtained, and the consequences for the properties of quark stars are discussed in the next Section.

\section{RESULTS}

We use the same parametrization of the quark matter model as in [21]

$$
\begin{aligned}
m_{u, d} & =5.5 \mathrm{MeV}, \\
m_{s} & =112.0 \mathrm{MeV}, \\
G_{S} \Lambda^{2} & =2.319 \\
\Lambda & =602.3 \mathrm{MeV} .
\end{aligned}
$$

The relative diquark coupling strength, $\eta$, is considered as a free parameter of the model. Here we present results for $\eta=0.75$ (intermediate coupling), which is the vacuum result obtained by a Fierz transformation, and $\eta=1.0$ (strong coupling), which is motivated by the phenomenology of compact stars and heavy ion collisions [29]. Quark matter phases are characterized by the order parameters, $\phi_{i}$ and $\Delta_{i j}$. In particular, the following phases have been identified in the numerical investigation of the model

$$
\begin{aligned}
& \text { 2SC phase: } \Delta_{u s}=\Delta_{d s}=0 \text { and } \Delta_{u d} \neq 0, \\
& \text { uSC phase: } \Delta_{d s}=0 \text { and } \Delta_{u d} \neq 0, \Delta_{u s} \neq 0, \\
& \text { dSC phase: } \Delta_{u s}=0 \text { and } \Delta_{u d} \neq 0, \Delta_{d s} \neq 0, \\
& \text { CFL phase: } \Delta_{u d} \neq 0, \Delta_{u s} \neq 0, \text { and } \Delta_{d s} \neq 0 .
\end{aligned}
$$

In addition, gapless phases exist, which are characterized by the presence of one or more quasiparticle dispersion relations that have no forbidden energy band above the Fermi surface. Such excitations exist when the differences between the Fermi momenta and/or the renormalized masses of the paired quarks are sufficiently large [30]. We denote gapless phases with a leading "g", e.g., g2SC for the gapless 2SC phase.

In the following we present results for two different neutrino untrapping scenarios. In one scenario the initial state is a hot, $T=40 \mathrm{MeV}$, quark core with trapped neutrinos, $\mu_{\nu_{e}}=200 \mathrm{MeV}$. The core cools rapidly by neutrino emission and a cold, $T \sim 1 \mathrm{MeV}$, configuration with trapped neutrinos, $\mu_{\nu}=200 \mathrm{MeV}$, forms. The mass defect due to cooling is obtained by comparing the masses of these two states for configurations of equal baryon number. At low temperature, $T \sim 1 \mathrm{MeV}$, the mean-free path of neutrinos increases and becomes comparable to the size of the core/star. The neutrinos therefore escape and the final state is a cold, $T \sim 1 \mathrm{MeV}$, star with $\mu_{\nu}=0$. The mass defect due to neutrino untrapping is obtained by comparing the masses of $\mu_{\nu}=200 \mathrm{MeV}$, $T \sim 1 \mathrm{MeV}$ configurations with the masses of $\mu_{\nu}=0$, $T \sim 1 \mathrm{MeV}$ configurations of equal baryon number. The 
"final" state will continue to cool for millions of years with practically no effect on its structure, because temperatures below $1 \mathrm{MeV}$ have negligible effects on the quark matter equation of state. Phase diagrams for intermediate and strong coupling are provided for matter with trapped and untrapped neutrinos. The second scenario has a more "conventional" initial state, characterized by a fixed lepton number, $Y_{L_{e}}=0.4$, and entropy per baryon, $s / n_{B}=1-2$. In this case the temperature and neutrino chemical potential varies over the radius of the star. The properties of the quark star solutions and the effect of neutrino untrapping are similar in these two scenarios. While the initial state of the second scenario is motivated by detailed core-collapse simulations, it is more complicated and to some extent it provides less transparent results.

\section{A. Order parameters at fixed $\mu$}

For the calculation of quark matter phase diagrams in the plane of temperature and quark (or baryon) number chemical potential, the gap equations for the order parameters of the model (the masses, $M_{i}$, and diquark gaps, $\left.\Delta_{i j}\right)$ are solved self-consistently. The values of the order parameters characterize the different quark matter phases, as described above. In addition to solving the gap equations, the color and electric charge neutrality conditions are enforced by solving for the corresponding chemical potentials $\left(\mu_{Q}, \mu_{3}, \mu_{8}\right)$, for given values of the quark number chemical potential, $\mu$, and temperature, $T$. In Figs. 1,4, the quark masses (upper row), diquark gaps (second row), chemical potentials (third row) and densities of baryon number, electrons and muons (bottom row) are plotted vs. the temperature for three different values of the quark number chemical potential (columns). The four Figures represent solutions without $\left(\mu_{\nu}=0\right)$ and with $\left(\mu_{\nu}=200 \mathrm{MeV}\right)$ trapped neutrinos for intermediate $(\eta=0.75)$ and strong $(\eta=1.0)$ coupling, respectively. Here we use the more compact notation

$$
\mu_{\nu} \equiv \mu_{\nu_{e}}=\mu_{L_{e}}
$$

for the electron neutrino chemical potential, because the muon neutrino chemical potential is zero as neutrino oscillations are neglected. Note the gapless constraints, $\Delta_{i j}^{g}$, that are included in the second row of these Figures. If $\Delta_{i j} \leq \Delta_{i j}^{g}$, the corresponding quasiparticle has a gapless dispersion relation, i.e., when this condition is met there is no forbidden energy band above the Fermi surface. Typically, the gapless conditions are fulfilled only near the critical temperature $(60-70 \mathrm{MeV}$ for intermediate coupling and $100-110 \mathrm{MeV}$ for strong coupling), where the gaps go to zero in a second order phase transition to a non-superconducting state. Since these temperatures are well above the maximum temperature relevant for PNS, we can neglect gapless phases in our discussion.

\section{B. Order parameters at fixed $Y_{L_{e}}$ and $s / n_{B}$}

Next we consider a system with fixed values of the lepton fraction, $Y_{L_{e}}\left(T, \mu_{\nu}\right)=0.4$, and the entropy per baryon, $s\left(T, \mu_{\nu}\right) / n_{B}\left(T, \mu_{\nu}\right)=1,2$. Consequently, for a given value of the quark number chemical potential, $\mu$, the temperature and electron neutrino chemical potential are determined such that $Y_{L_{e}}=0.4$ and $s / n_{B}=1$ (or 2 ). These two equations are solved in parallel with the gap and charge neutrality equations. In Fig. 5 the temperature, constituent quark masses, charge chemical potentials, neutrino chemical potential, gaps, and number densities are plotted vs. the quark number chemical potential for charge neutral quark matter in $\beta$-equilibrium at intermediate coupling, $\eta=0.75$. Fig. 6 shows the same relationships for strong coupling, $\eta=1$.

\section{Equations of state}

The equation of state (EoS), $\epsilon(P)$, can be calculated using (16), e.g., for different values of the temperature, $T$, and lepton number chemical potential, $\mu_{L_{e}}=\mu_{\nu}$, or for fixed values of lepton fraction, $Y_{L_{e}}\left(T, \mu_{L_{e}}\right)$, and the entropy per baryon, $s\left(T, \mu_{L_{e}}\right) / n_{B}\left(T, \mu_{L_{e}}\right)$, depending on the application. In Figs. 7,8 the EoS for three different pairs of $\left(\mu_{\nu}, T\right)$ values are plotted for intermediate, $\eta=$ 0.75 , and strong, $\eta=1.0$, coupling, respectively. The energy density is discontinuous at the 2SC-CFL phase transition due to the discontinuity of the strange quark mass, which is obtained from the self-consistent solution of the system of gap equations (15). The phase transition is first order. When increasing $T$ and/or $\mu_{\nu}$, the pressure at the transition increases. Fig. 9 shows the EoS for a fixed lepton fraction, $Y_{L_{e}}\left(T, \mu_{\nu}\right)=0.4$, and two values of entropy per baryon, $s\left(T, \mu_{\nu}\right) / n_{B}\left(T, \mu_{\nu}\right)=1$ and 2 , for intermediate and strong coupling. When increasing the entropy per baryon, the pressure at the transition increases, whereas an increase of the coupling strength lowers it.

\section{Quark star sequences}

Given an EoS described in the previous Section, the corresponding compact star sequence is calculated by solving the Tolman-Oppenheimer-Volkoff equations for a static spherically symmetric object

$$
\begin{aligned}
\frac{d P(r)}{d r} & =-\frac{[\epsilon(r)+P(r)]\left[m(r)+4 \pi r^{3} P(r)\right]}{r[r-2 m(r)]} \\
m(r) & =4 \pi \int_{0}^{r} \varepsilon\left(r^{\prime}\right) r^{\prime 2} d r^{\prime}
\end{aligned}
$$

for different values of the central pressure. Due to the thermal pressure, the high-temperature EsoS do not extend to zero pressure. The surface of hot configurations is therefore defined to be the point where the (approxi- 

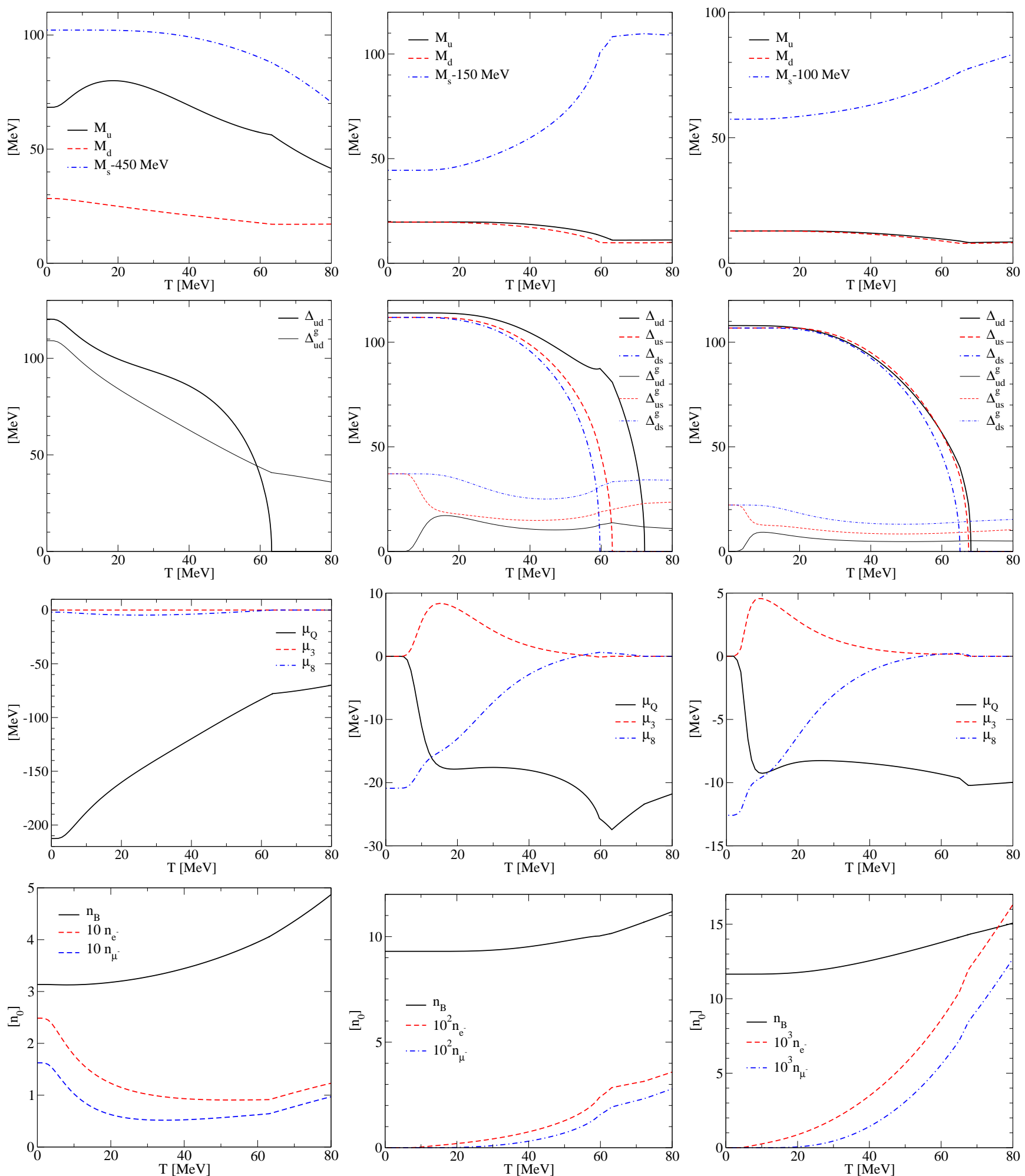

FIG. 1: (Color online) The constituent quark masses, gaps, charge chemical potentials, and number densities of charge neutral quark matter in $\beta$-equilibrium with untrapped neutrinos, $\mu_{\nu}=0$, at intermediate coupling, $\eta=0.75$. The three columns (from left to right) represent solutions for $\mu=400,500$, and $550 \mathrm{MeV} . \Delta_{i j}^{g}$ are thresholds for the existence of gapless excitations [30], i.e., gapless excitations exist if $\Delta_{i j} \leq \Delta_{i j}^{g}$. 

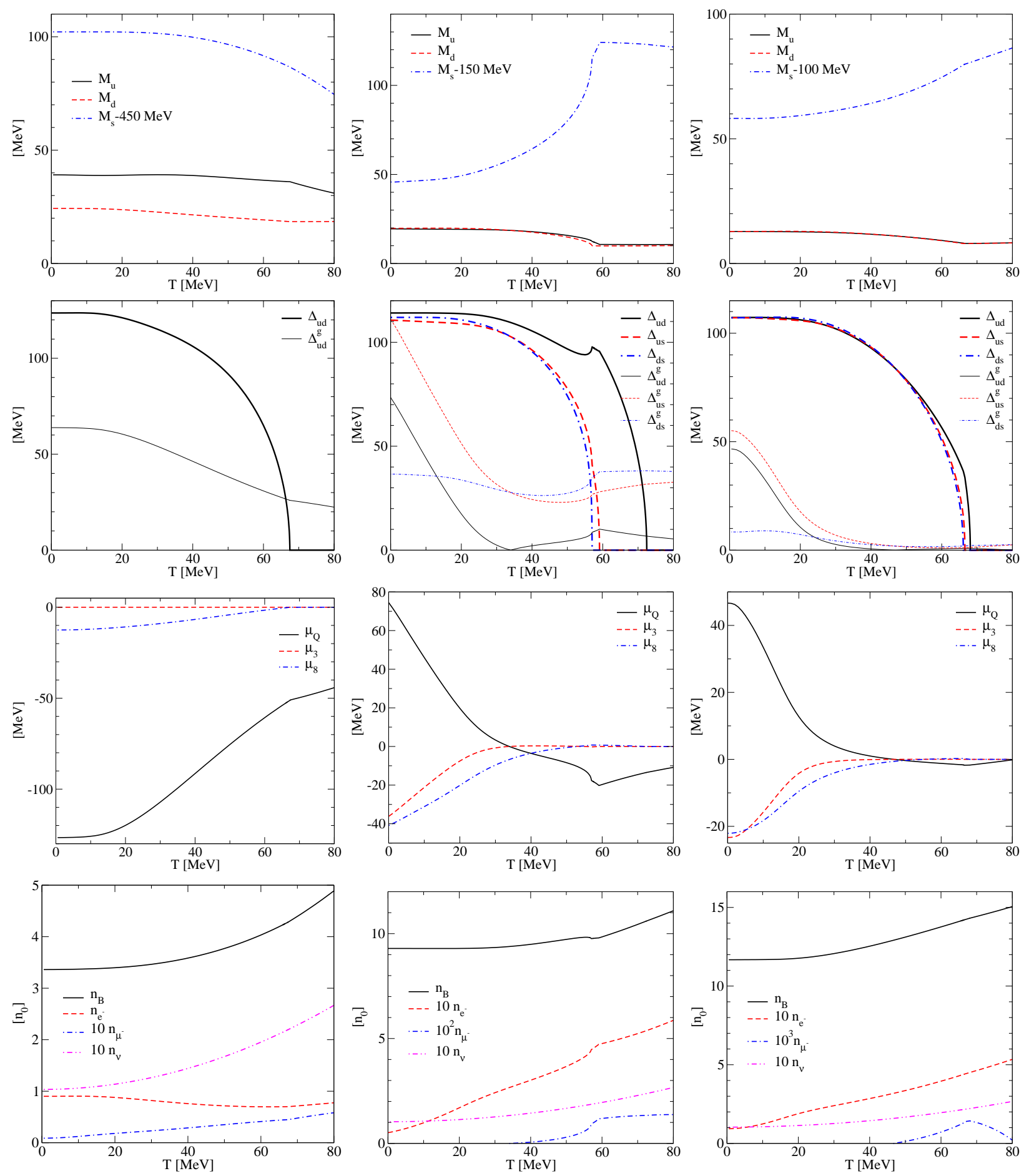

FIG. 2: (Color online) The constituent quark masses, gaps, charge chemical potentials, and number densities of charge neutral quark matter in $\beta$-equilibrium with trapped neutrinos, $\mu_{\nu}=200 \mathrm{MeV}$, at intermediate coupling, $\eta=0.75$. Line styles as in Fig. 1. 

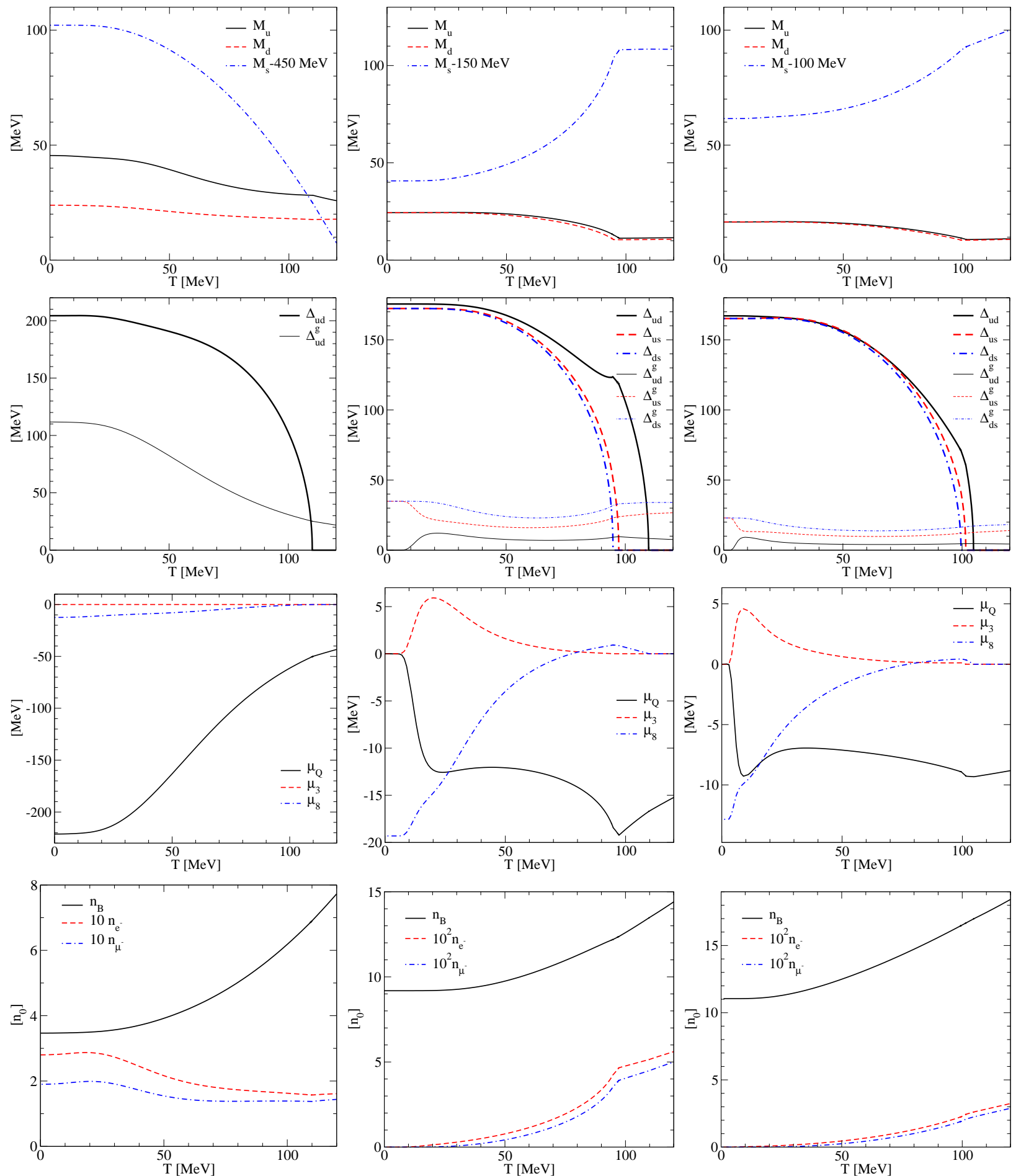

FIG. 3: (Color online) The constituent quark masses, gaps, charge chemical potentials, and number densities of charge neutral quark matter in $\beta$-equilibrium with untrapped neutrinos, $\mu_{\nu}=0$, at strong coupling, $\eta=1.0$. Line styles as in Fig. 11. 

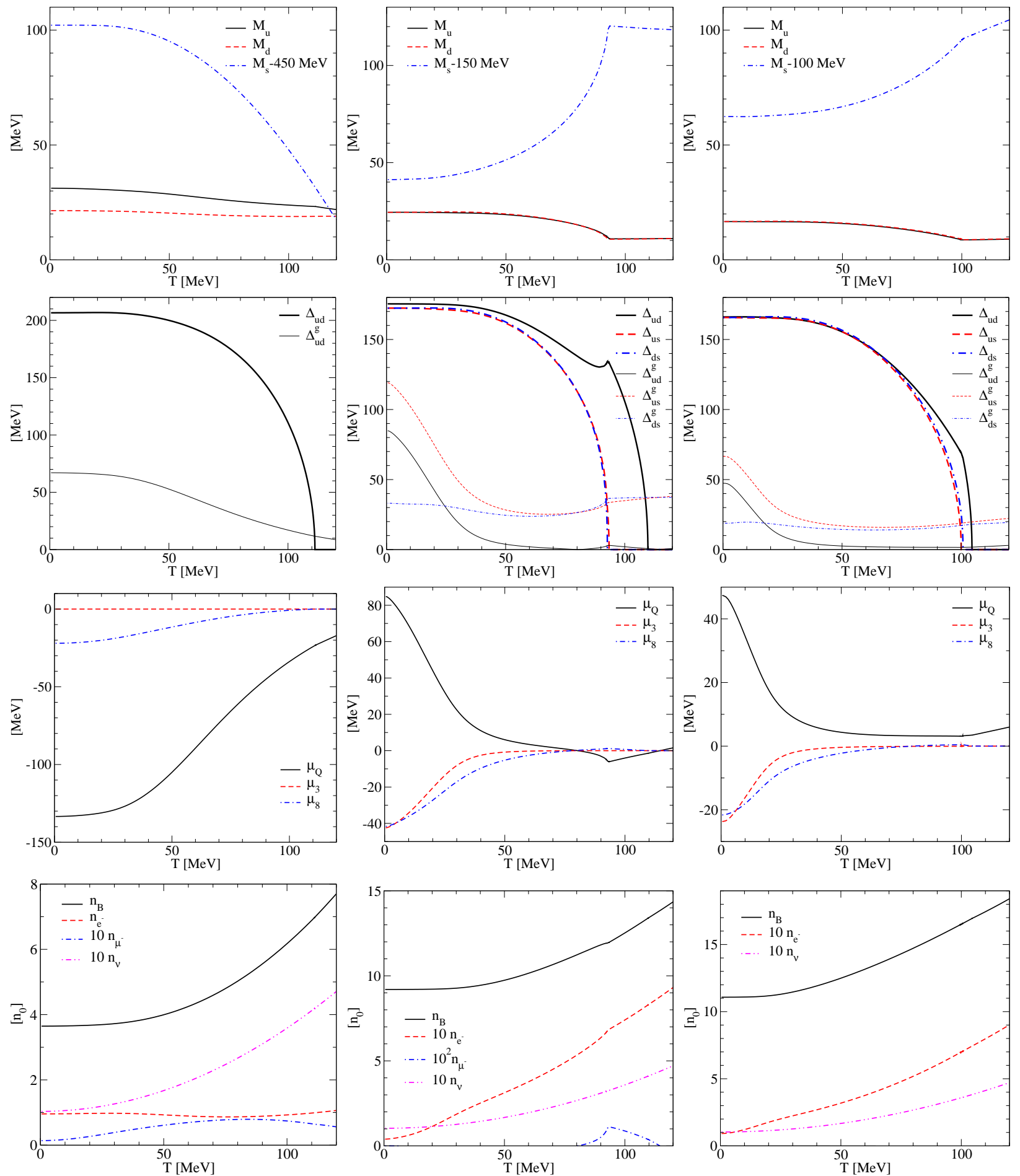

FIG. 4: (Color online) The constituent quark masses, gaps, charge chemical potentials, and number densities of charge neutral quark matter in $\beta$-equilibrium with trapped neutrinos, $\mu_{\nu}=200 \mathrm{MeV}$, at strong coupling, $\eta=1.0$. Line styles as in Fig. 1. 

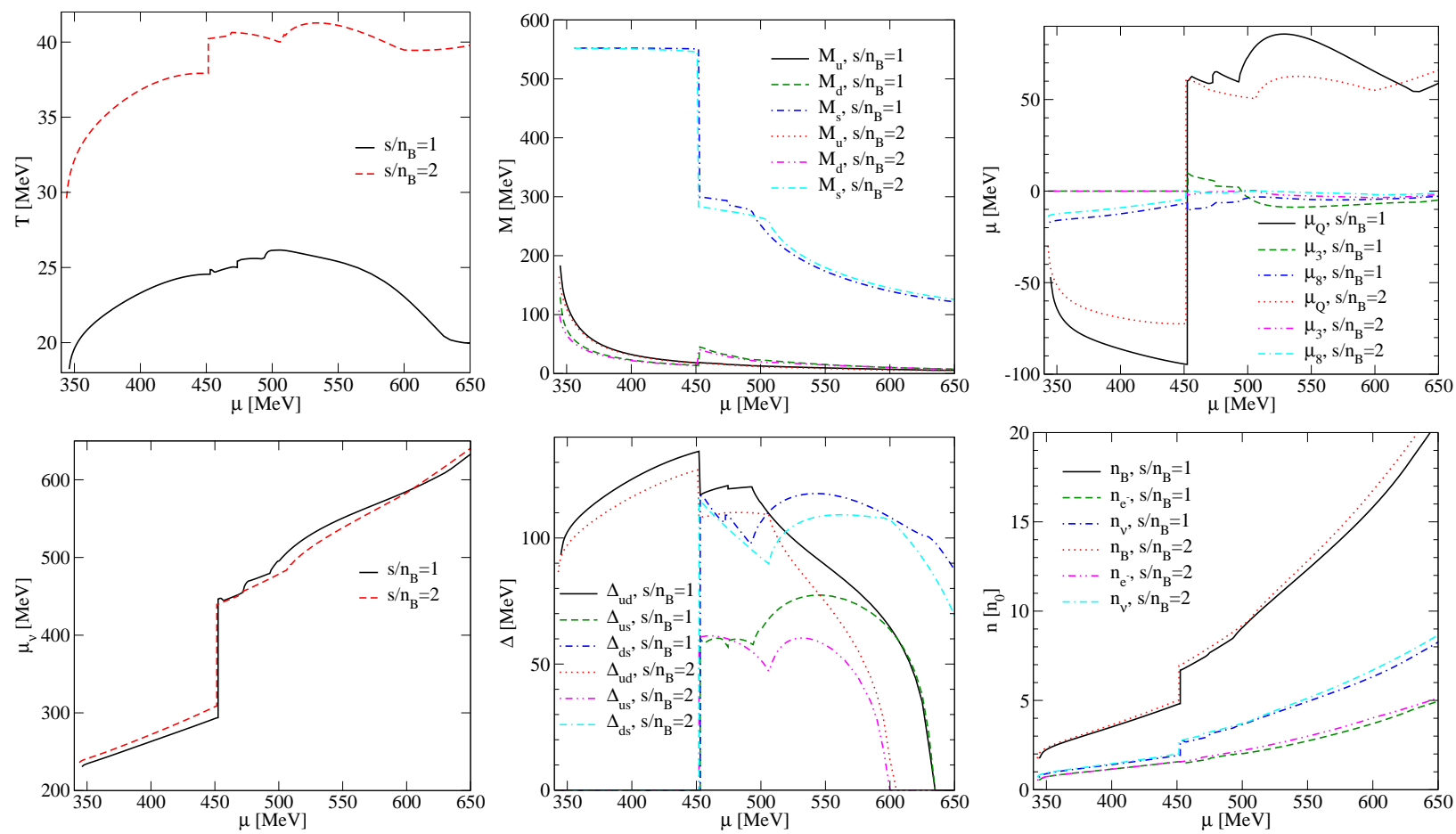

FIG. 5: (Color online) The temperature, constituent quark masses, charge chemical potentials, neutrino chemical potential, gaps, and number densities of charge neutral quark matter in $\beta$-equilibrium at intermediate coupling $(\eta=0.75)$ for fixed lepton fraction $Y_{L_{e}}\left(T, \mu_{\nu}\right)=0.4$, and two values of the entropy per baryon, $s\left(T, \mu_{\nu}\right) / n_{B}\left(T, \mu_{\nu}\right)=1,2$.
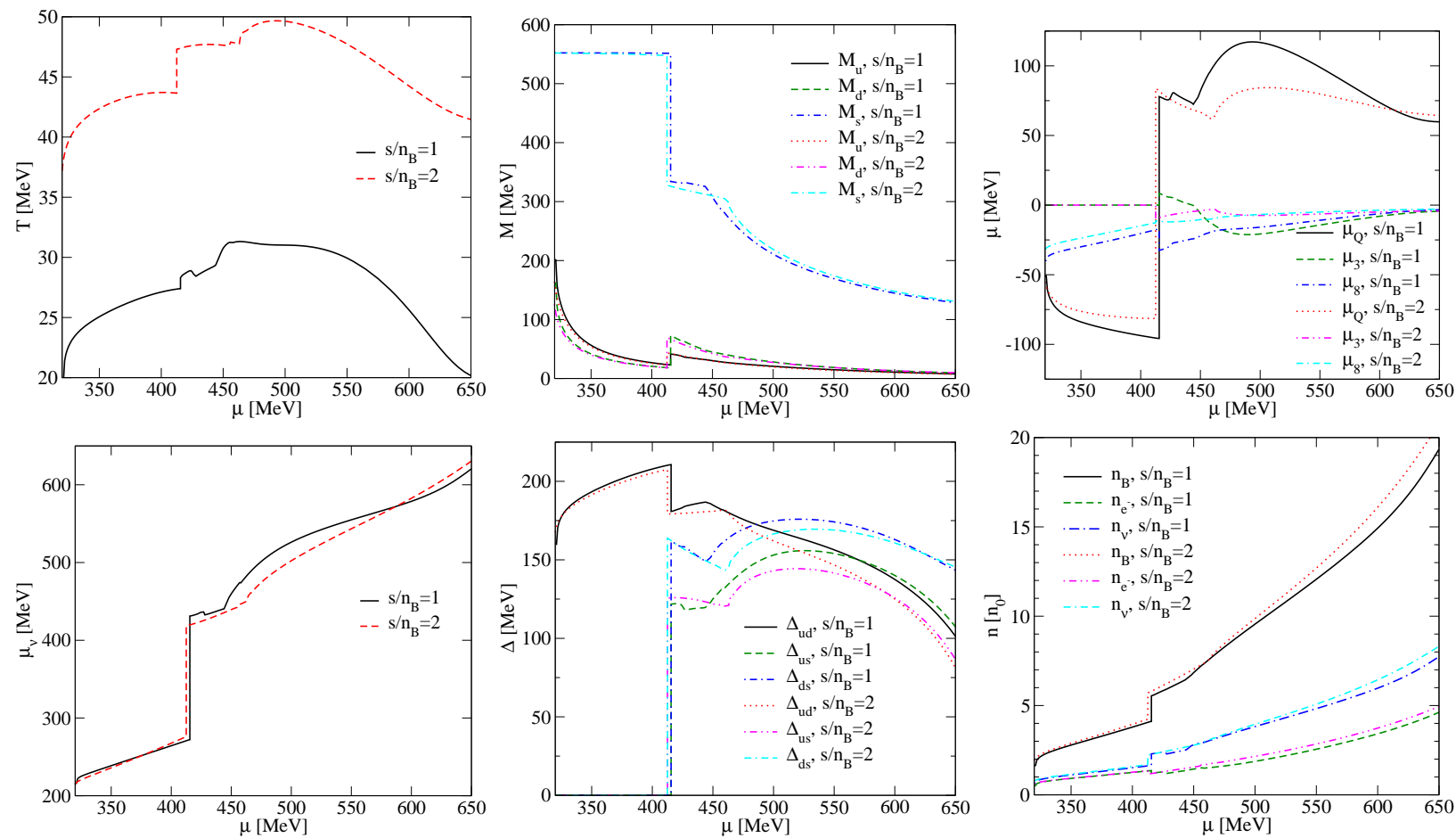

FIG. 6: (Color online) The same as Fig. 5 but at strong coupling, $\eta=1$. 


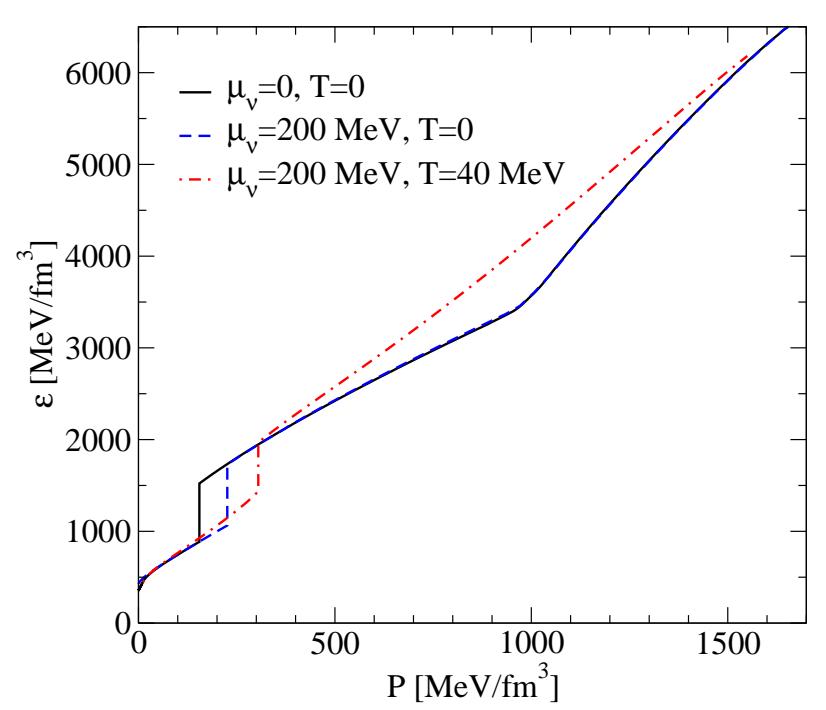

FIG. 7: (Color online) The equation of state for charge neutral quark matter in $\beta$-equilibrium at intermediate coupling, $\eta=0.75$, and fixed values of the temperature and electron neutrino chemical potential. The discontinuities appear at the 2SC-CFL phase transition due to the Maxwell construction, see text.

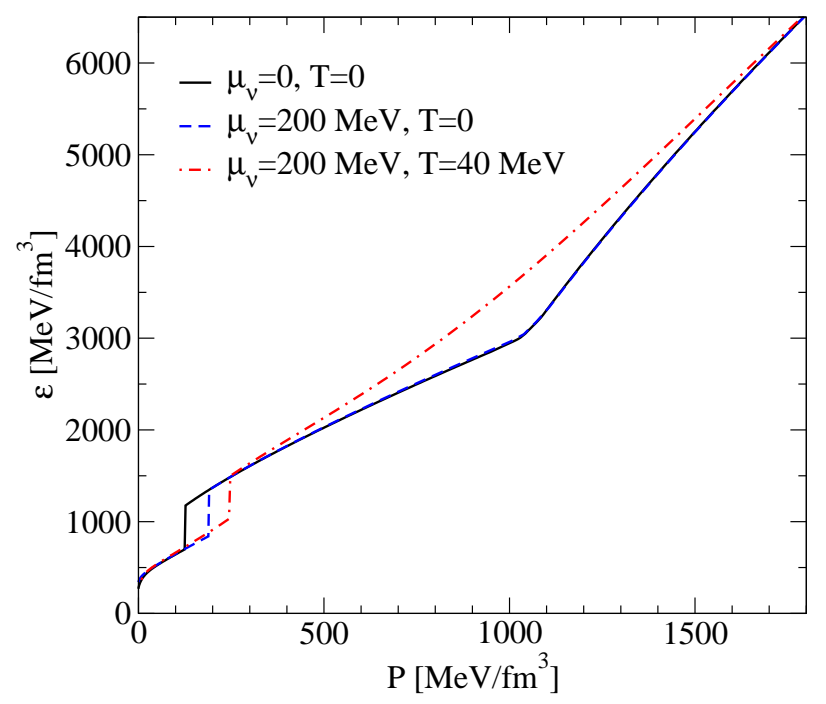

FIG. 8: (Color online) The equation of state for charge neutral quark matter in $\beta$-equilibrium at strong coupling, $\eta=1.0$, and fixed values of the temperature and electron neutrino chemical potential.

mate) chiral symmetry is broken by a first order phase transition into the $\chi \mathrm{SB}$ phase.

In Fig. 10 the quark star sequences for intermediate coupling, $\eta=0.75$, and fixed $\left(\mu_{\nu}, T\right)$ values are plotted. The discontinuity at the maximum mass configuration is a consequence of the appearance of CFL matter in the center of the stars. Stars with a CFL core are marginally stable and exist if $T$ and $\mu_{\nu}$ are not too high, see Fig. 14 in the following Subsection. Increasing the coupling to $\eta=1.0$ leads to an interesting situation, shown in Fig.11.

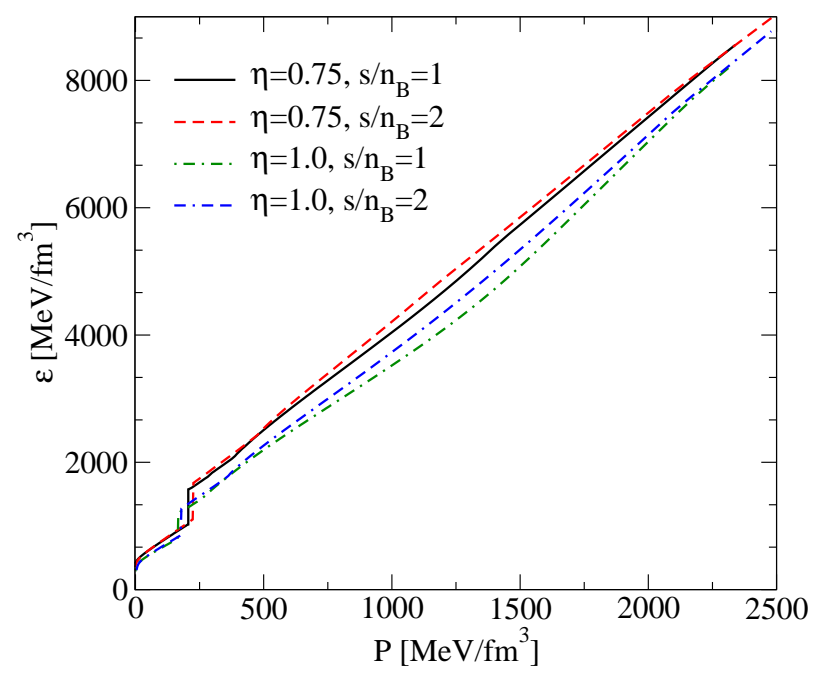

FIG. 9: (Color online) The equation of state for charge neutral quark matter in $\beta$-equilibrium for fixed values of the lepton fraction, $Y_{L_{e}}\left(T, \mu_{\nu}\right)=0.4$, and the entropy per baryon, $s\left(T, \mu_{\nu}\right) / n_{B}\left(T, \mu_{\nu}\right)$.

There is a small interval of star masses at $M \sim 1.3 \mathrm{M}_{\odot}$ for which mass twins occur: pure 2SC quark stars have stable, high-density mass isomers with a CFL quark core and a smaller radius. Fig. 11] suggests an interesting scenario: upon mass accretion, a 2SC star could undergo a phase transition to its more compact twin with a CFL core, whereby binding energy is released. However, the presence of a hadronic crust could render the twin configurations unstable and this scenario therefore remains to be investigated thoroughly. In Fig. 12 we show the star sequences corresponding to the EsoS in Fig. 9 with fixed entropy per baryon (1 or 2) and fixed lepton fraction $Y_{L_{e}}=0.4$. While the increase of coupling strength increases the maximum mass and the radius of the stars, both values of the entropy per baryon produce rather similar star sequences. A finite entropy per baryon of $1-2$ correspond to rather high temperatures that do not allow for stable CFL cores. In Fig. 13 the radial dependence of the temperature and baryon number density in PNS is illustrated for fixed values of the lepton fraction and entropy per baryon. These configurations, which resemble realistic PNS formed in the adiabatic compression of the cores in massive stars, have approximately constant temperature and neutrino chemical potential in their interior. Consequently, at the qualitative level the initial state of a PNS can be modeled by a fixed initial temperature, $T(r)=T$, and neutrino chemical potential, $\mu_{\nu}(r)=\mu_{\nu}$. This result justifies the alternative neutrino trapping/untrapping scenario considered in this paper.

\section{E. Phase diagrams}

Next we present for the first time the central temperatures and quark number chemical potentials of stable 


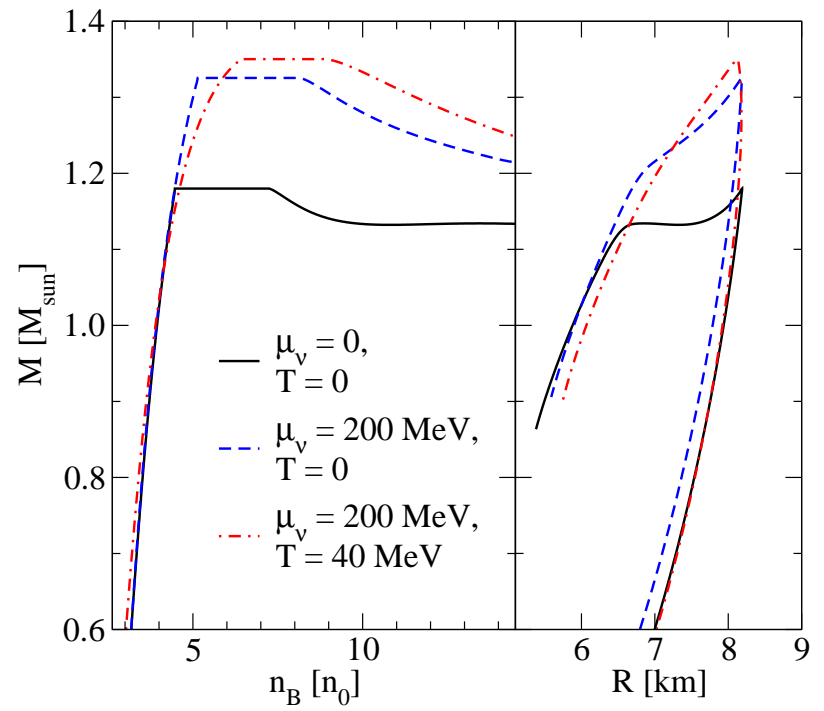

FIG. 10: (Color online) Quark star sequences for intermediate coupling, $\eta=0.75$, and fixed values of the temperature and electron neutrino chemical potential. The discontinuity at the maximum mass configuration is a consequence of the appearance of CFL matter in the center of the stars. Stars with a CFL core are marginally stable and exist if the temperature and neutrino chemical potential are not too high, see Fig. 14.

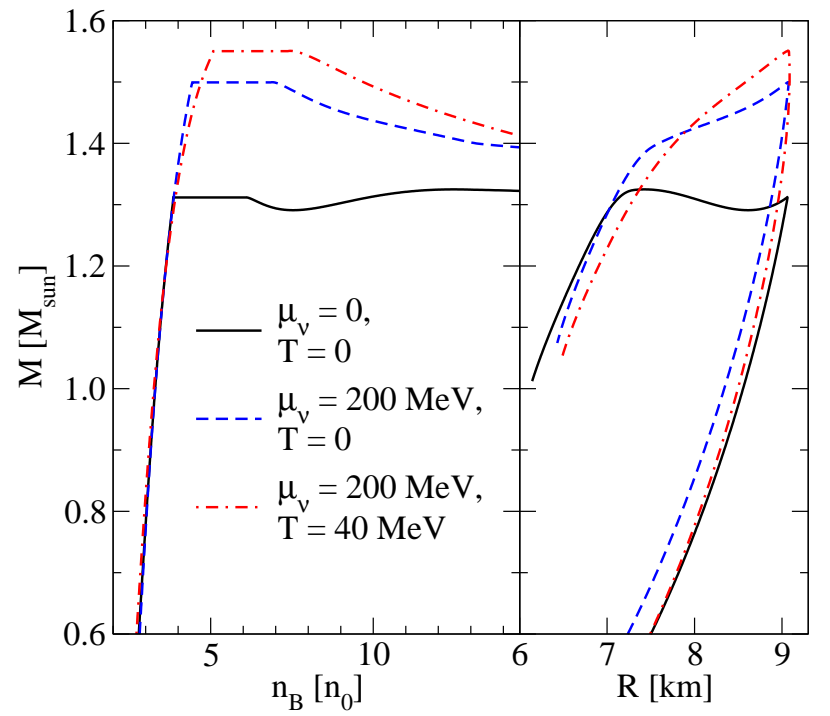

FIG. 11: (Color online) Quark star sequences for the strong coupling, $\eta=1.0$, and fixed values of the temperature and electron neutrino chemical potential. Stable stars with a CFL core exist if the temperature and neutrino chemical potential are not too high, see Fig. 15.

quark star solutions in the phase diagrams of quark matter, with and without neutrino trapping. In Figs. 14. 15] the phase diagrams of charge neutral quark matter in $\beta$-equilibrium are shown for intermediate, $\eta=0.75$, and strong, $\eta=1$, coupling and for two different values of the electron neutrino chemical potential, $\mu_{\nu}=0$ and $200 \mathrm{MeV}$.

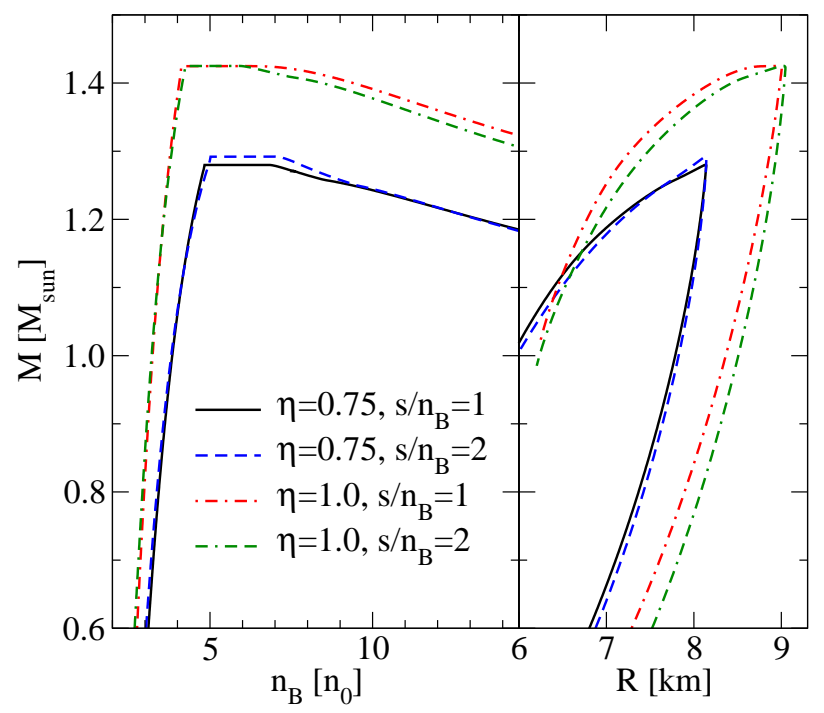

FIG. 12: (Color online) Quark star sequences for fixed values of the lepton fraction, $Y_{L_{e}}\left(T, \mu_{\nu}\right)=0.4$, and the entropy per baryon, $s\left(T, \mu_{\nu}\right) / n_{B}\left(T, \mu_{\nu}\right)$. Stars with a CFL core are unstable (marginally unstable for $\eta=1$ ).

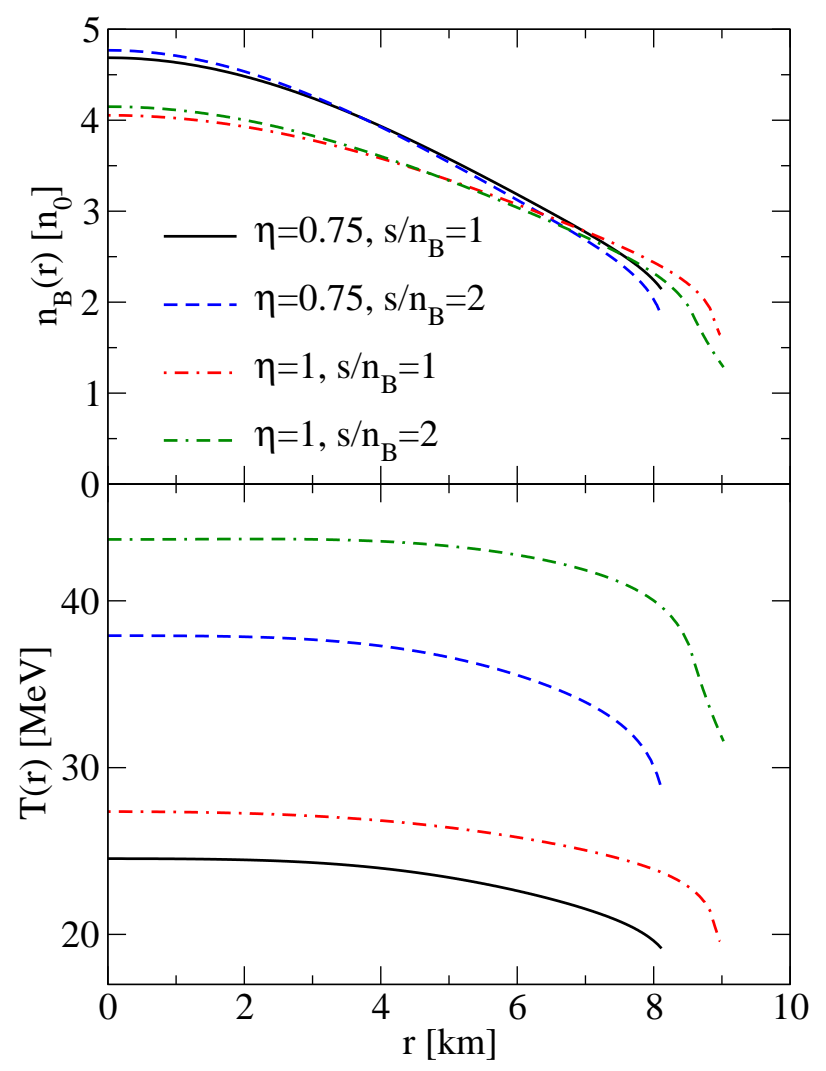

FIG. 13: (Color online) The temperature and baryon number density vs. the coordinate radius of four different quark star solutions, for fixed values of the lepton fraction, $Y_{L_{e}}\left(T, \mu_{\nu}\right)=$ 0.4 , and the entropy per baryon, $s\left(T, \mu_{\nu}\right) / n_{B}\left(T, \mu_{\nu}\right)$. The mass of these quark star solutions is $1.25 \mathrm{M}_{\odot}\left(1.4 \mathrm{M}_{\odot}\right)$ for intermediate (strong) coupling. 

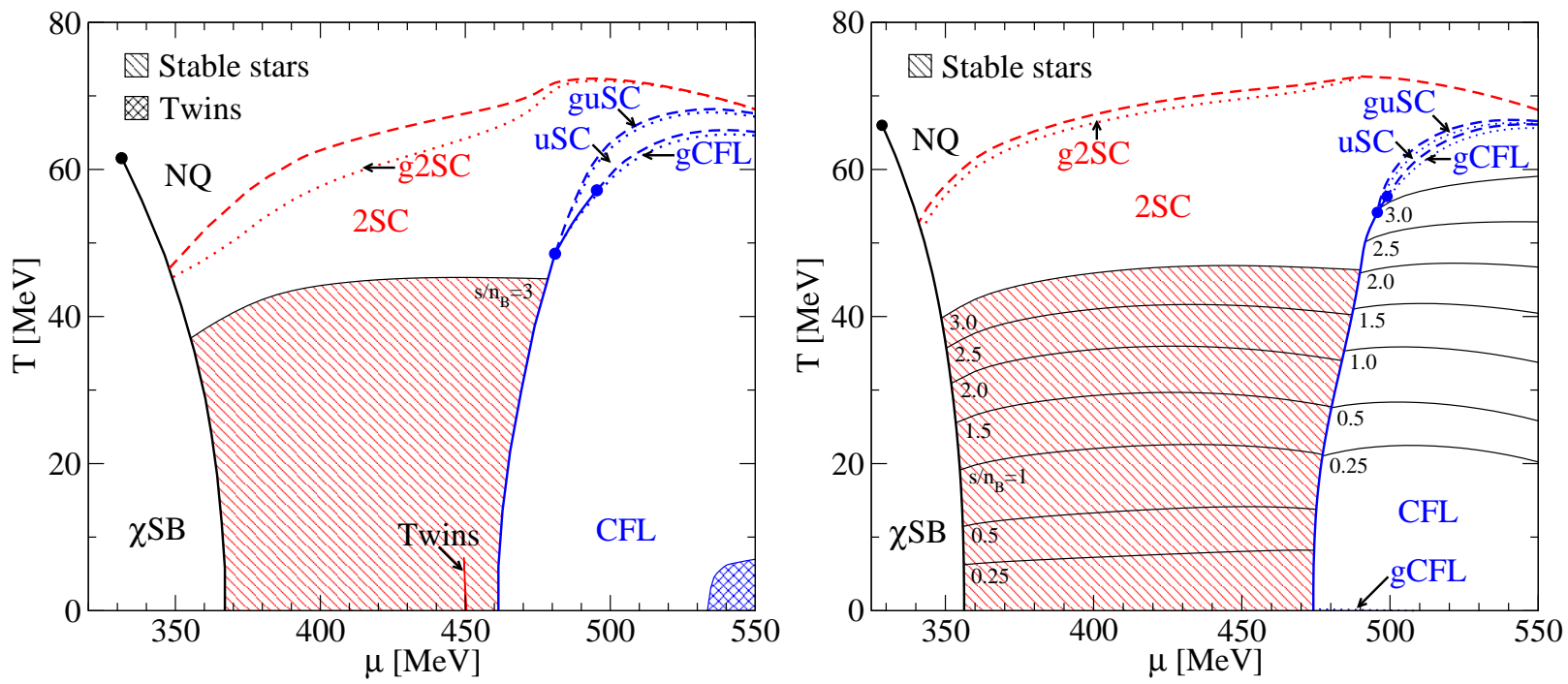

FIG. 14: (Color online) Phase diagrams of charge neutral quark matter in $\beta$-equilibrium at intermediate coupling, $\eta=0.75$, for fixed values of the electron neutrino chemical potential, $\mu_{\nu}=0$ (left-hand side) and $\mu_{\nu}=200 \mathrm{MeV}$ (right-hand side). First-order phase transition boundaries are indicated by bold solid lines, while bold dashed lines represent second-order phase boundaries. The dotted lines indicate gapless phase boundaries and the thin solid lines are level curves of constant entropy per baryon. Hatched regions represent stable compact star solutions, with central quark number chemical potential $\mu(r=0)=\mu$ and temperature $T(r=0)=T$. The cross-hatched regions correspond to baryon number twins, i.e., for these values of $\mu(r=0)$ and $T(r=0)$ there exist stable 2SC stars and 2SC-CFL stars with equal baryon number.
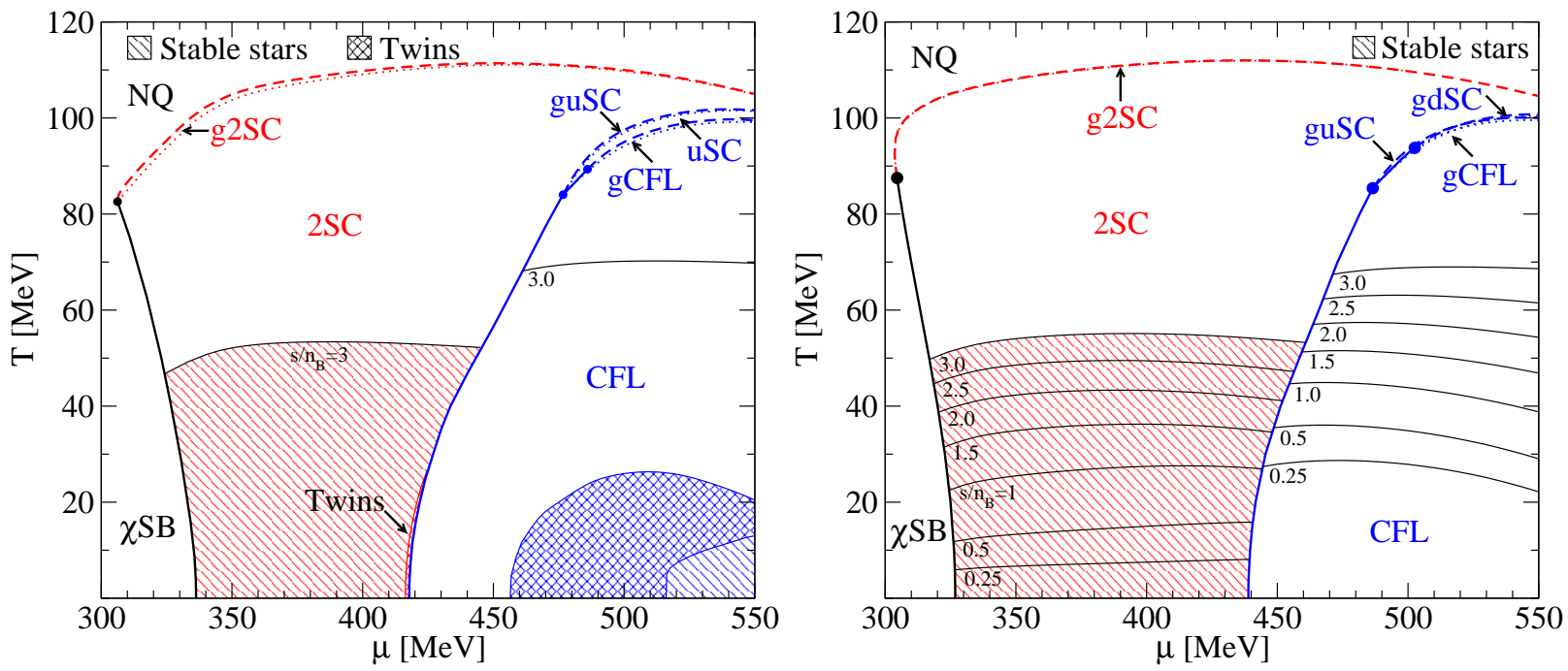

FIG. 15: (Color online) Phase diagrams of charge neutral quark matter in $\beta$-equilibrium at strong coupling, $\eta=1.0$, for fixed values of the electron neutrino chemical potential, $\mu_{\nu}=0$ (left-hand side) and $\mu_{\nu}=200 \mathrm{MeV}$ (right-hand side). Line styles as in Fig. 14 


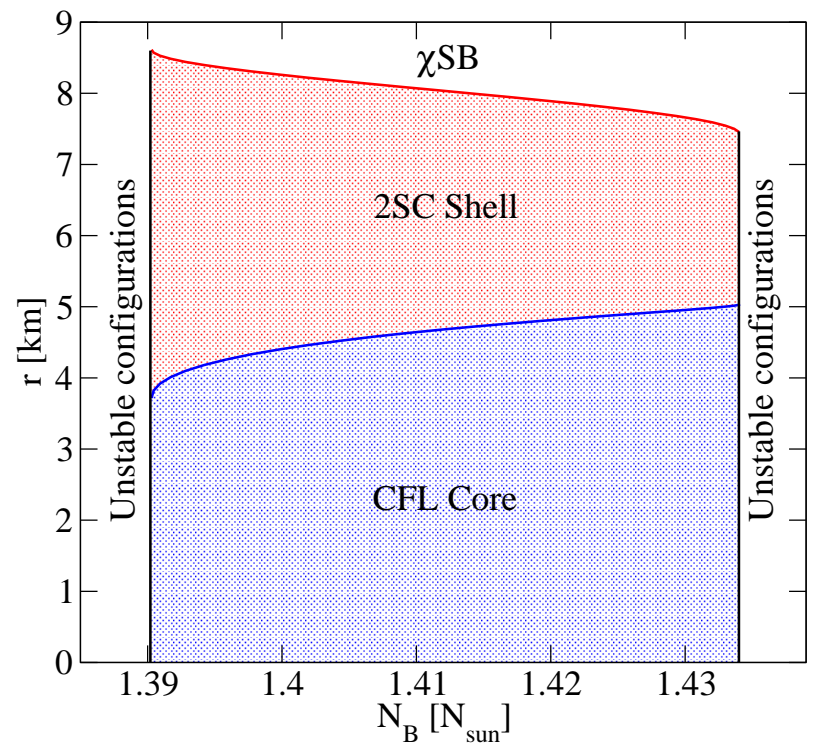

FIG. 16: (Color online) Radial phase structure of final states with CFL cores at strong coupling, $\eta=1.0$. The $T=0$, $\mu_{\nu}=0$ compact star sequence in Fig. 11] has a branch of stable stars with CFL cores, this Figure shows the radial phase structure of these stars.

As an upper limit estimate for the initial core temperatures of quark stars we show the lines corresponding to an entropy per baryon of 3 , which in the $2 \mathrm{SC}$ phase leads to a maximum temperature of $\sim 40 \mathrm{MeV}(\sim 50 \mathrm{MeV})$ for intermediate (strong) coupling. For sufficiently high neutrino chemical potentials, quark stars with a CFL core are unstable. In particular, by comparing the lefthand $\left(\mu_{\nu}=0\right)$ and right-hand $\left(\mu_{\nu}=200 \mathrm{MeV}\right)$ panels of Fig. 15]it is clear that stars with a CFL core are rendered unstable by the finite neutrino chemical potential. For a typical value of $\mu_{\nu}=200 \mathrm{MeV}$, which should be realized in the evolution of a PNS core [3, 4], we find no stable CFL cores. Note that the discussion of star temperatures exceeding the neutrino opacity temperature, $T_{c} \sim 1 \mathrm{MeV}$, makes sense only during the PNS era, i.e., when neutrinos are trapped and the neutrino chemical potential is finite. Consequently, the region of stable CFL core stars shown in the panel on the left-hand side of Fig. 15 may not be realized at all during stages of hot PNS (quark star) evolution. We will return to this issue in the next Subsection.

For temperatures below $T_{c}$, we find a range of baryon numbers $N_{B}=1.39-1.43(5) \mathrm{N}_{\odot}$, for which quark stars with a CFL core and a $2 \mathrm{SC}$ shell are stable. The phase structure of these stars is illustrated in Fig. 16. Gapless phases of superconducting quark matter exist for both coupling strengths, at temperatures well above those of relevance for the cores of PNS, see Figs. 14][15, The gapless phases can therefore be neglected in the present discussion of PNS evolution [31].

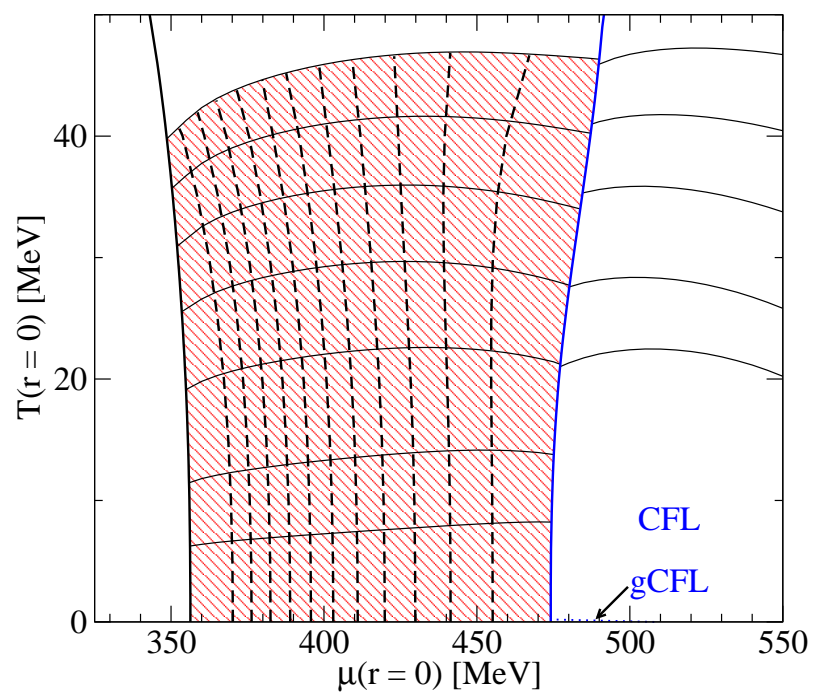

FIG. 17: (Color online) Cooling of PNS with trapped neutrinos, $\mu_{\nu}=200 \mathrm{MeV}$, at intermediate coupling, $\eta=0.75$. The dashed lines indicate the central temperature and quark number chemical potential of configurations with fixed baryon number, $N=0.2,0.3,0.4, \ldots, 1.2 \mathrm{~N}_{\odot}$. Solid lines indicate phase boundaries and level curves of constant entropy per baryon, see Fig. 14]

\section{F. Cooling evolution and untrapping transition}

In this Subsection we consider a scenario of quark star cooling where the baryon number of the stars is conserved, i.e., we assume that there are neither accretion nor mass loss. In Figs. 17]18 we show the cooling evolution of PNS configurations in the phase diagrams of quark matter with trapped neutrinos, $\mu_{\nu}=200 \mathrm{MeV}$, for intermediate and strong coupling. The dashed lines indicate the central temperature and quark number chemical potential of configurations with fixed baryon number. The vertical solid lines indicate phase boundaries of the $2 \mathrm{SC}$ phase, while the horizontal lines denote curves of constant entropy per baryon. We observe that, as long as the neutrino chemical potential remains fixed, the cooling trajectories that start from stable configurations remain inside the region of stability. The reverse, however, does not hold. Upon heating, a configuration close to the CFL phase border may become unstable at high temperature. See, e.g., the trajectory for $N=1.6 \mathrm{~N}_{\odot}$ in Fig. 18,

Another instability occurs during the cooling evolution when a star reaches the neutrino opacity temperature, $T_{c} \sim 1 \mathrm{MeV}$, and neutrinos start leaving the system. The microscopic processes behind this neutrino untrapping transition are elastic and inelastic neutrino-quark collisions, which lead to a neutrino mean free path that exceeds the radius of the star as the temperature decreases to $T_{c}$. This characterizes the transition from the neutrino-diffusion regime with finite $\mu_{\nu}$ to the freestreaming regime with $\mu_{\nu}=0$, which takes place within the transport timescale of a few milliseconds [18]. Since 


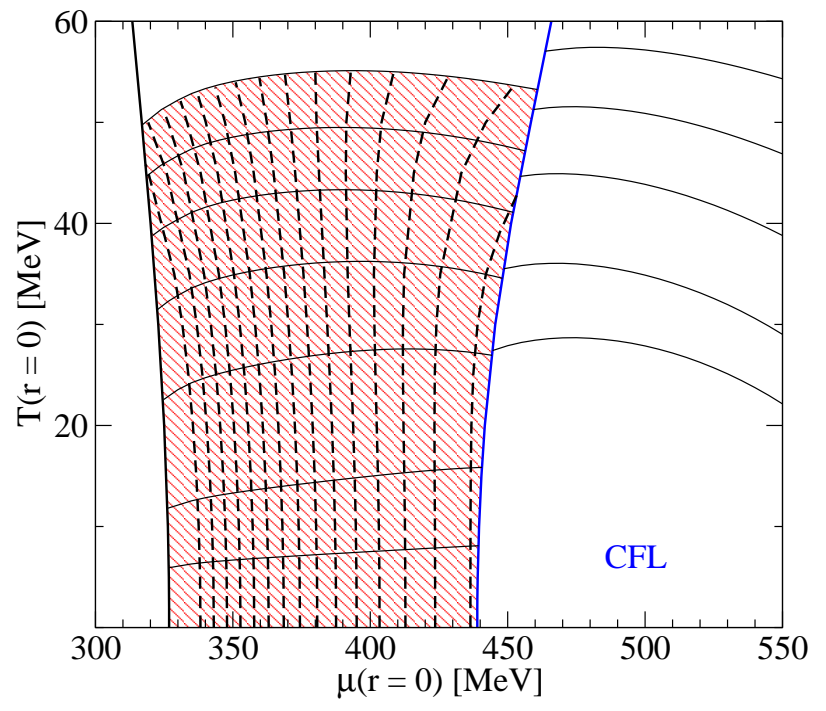

FIG. 18: (Color online) Cooling of PNS with trapped neutrinos, $\mu_{\nu}=200 \mathrm{MeV}$, at strong coupling, $\eta=1.0$. The dashed lines indicate the central temperature and quark number chemical potential of configurations with fixed baryon number, $N=0.2,0.3,0.4, \ldots, 1.6 \mathrm{~N} \odot$. Solid lines indicate phase boundaries and level curves of constant entropy per baryon, see Fig. 15.

this timescale is well above the strong and weak interaction timescales, which establish the local thermal and chemical equilibrium, the untrapping transition can be considered as a quasi-static process that connects the initial state with finite neutrino chemical potential and the final state with vanishing $\mu_{\nu}$ by a sequence of equilibrium star configurations. This justifies the estimate of the energy release due to neutrino untrapping from the mass defect between initial and final configurations. Cooling trajectories that are close to the CFL phase border above $T_{c}$ continue within the CFL-twin region below it, i.e., conservation of baryon number requires that the phase structure of these stars change near $T_{c}$. Leaving aside the details of the untrapping transition itself, we illustrate this situation in Figs. 19] 20 for strong and intermediate coupling, respectively. In these figures, the phase diagram above (below) $T_{c}$ represents quark matter with trapped (untrapped) neutrinos. We now discuss the strong coupling case illustrated in Fig. 19 in more detail. At $T_{c}$ the mean free path of the neutrinos is similar to the size of the stars and $\mu_{\nu} \rightarrow 0$. The central quark (or baryon) number chemical potential of configurations with fixed baryon number increases in the untrapping transition. When the neutrinos have escaped there are stable $2 \mathrm{SC}$ stars for $N \lesssim 1.41 \mathrm{~N}_{\odot}$ and stable stars with a CFL core for $1.39 \mathrm{~N}_{\odot} \lesssim N \lesssim 1.43 \mathrm{~N}_{\odot}$. The dashed lines in the CFL phase indicate the central temperature and quark number chemical potential of configurations with fixed baryon number, $N=1.4,1.41,1.42 \mathrm{~N}_{\odot}$. The configurations with $N=1.4$ and $1.41 \mathrm{~N}_{\odot}$ are CFL baryon number twins, i.e., for these baryon numbers there exist

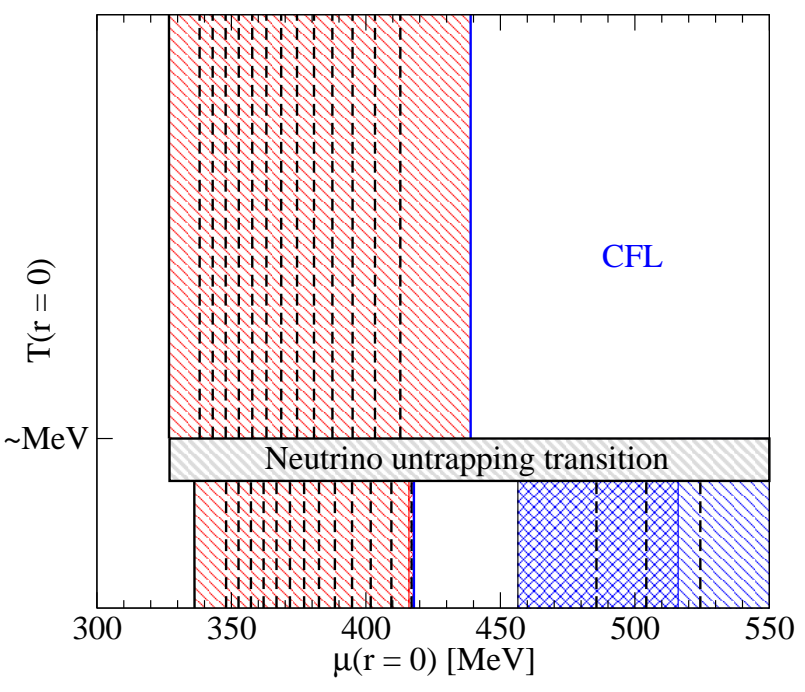

FIG. 19: (Color online) Neutrino untrapping transition at strong coupling, $\eta=1.0$. The dashed lines in the $2 \mathrm{SC}$ phase (See Fig. 15) indicate the central temperature and quark number chemical potential of configurations with fixed baryon number, $N=0.2,0.3,0.4, \ldots, 1.4 \mathrm{~N}_{\odot}$. Above the critical temperature, $T_{c} \sim 1 \mathrm{MeV}$, of the neutrino untrapping transition the neutrino mean free path is shorter than the size of the stars and $\mu_{\nu}$ is therefore fixed at $200 \mathrm{MeV}$. This corresponds to cold PNS with trapped neutrinos. At $T_{c}$ the mean free path of the neutrinos is similar to the size of the stars and $\mu_{\nu} \rightarrow 0$. The central baryon number chemical potential of configurations with fixed baryon number increases in the untrapping transition. When the neutrinos have escaped there are stable $2 \mathrm{SC}$ stars for $N \lesssim 1.41 \mathrm{~N}_{\odot}$ and stable stars with a CFL core for $1.39 \mathrm{~N}_{\odot} \lesssim N \lesssim 1.43 \mathrm{~N}_{\odot}$. The dashed lines in the CFL phase indicate the central temperature and quark number chemical potential of configurations with fixed baryon number, $N=1.4,1.41,1.42 \mathrm{~N}_{\odot}$. The configurations with $N=1.4$ and $1.41 \mathrm{~N}_{\odot}$ are CFL baryon number twins, i.e., for these baryon numbers there exist also pure 2SC stars. See Fig. 15 and Fig. 21 for further information.

also pure 2SC stars.

For intermediate coupling, shown in Fig. 20, there is only a narrow interval of baryon numbers for which a transition to marginally stable CFL baryon number twins is possible during untrapping. These baryon numbers are not shown in that Figure.

\section{G. Mass defects due to cooling and neutrino untrapping}

The cooling evolution of a hot PNS from $T \sim 40 \mathrm{MeV}$ to $T_{c} \sim 1 \mathrm{MeV}$ and the subsequent neutrino untrapping transition entail significant changes to the EoS and the structure of the star. This results in a decrease of the gravitational mass. The mass defect, i.e., the difference of the gravitational masses before and after the cooling and/or untrapping transition, corresponds to the energy that is released from the star, predominantly by neutrino 


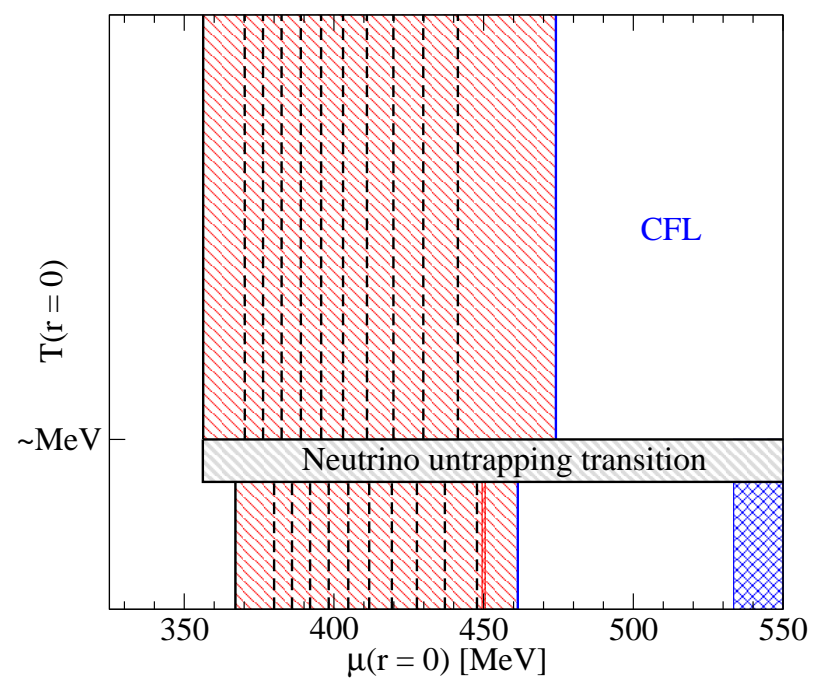

FIG. 20: (Color online) Neutrino untrapping transition at intermediate coupling, $\eta=0.75$. The dashed lines indicate the central temperature and quark number chemical potential of configurations with fixed baryon number, $N=0.2,0.3,0.4, \ldots, 1.1 \mathrm{~N}_{\odot}$. CFL baryon number twins are marginally stable and exist only for a narrow interval of baryon numbers. They are therefore omitted. For further information, see Fig. 14 and Fig. 19.

emission. A fraction of the neutrinos might, however, be converted into photons that give rise to a gamma- or Xray burst. The PNS cool rapidly by neutrino emission. A few seconds after birth the stars are cold on the nuclear scale $(T \sim 1 \mathrm{MeV})$ and further cooling has no direct impact on their structure. However, the neutrino mean-free path is sensitive to the temperature. Below some critical temperature $(\sim \mathrm{MeV})$ the neutrinos that are trapped in the PNS escape and this has an effect on the structure. In Figs. 21,22 the energy release is estimated from the mass defects due to cooling and neutrino untrapping for strong coupling and two different values of the baryon number.

In Figs. 23, 24 the energy release due to cooling and neutrino untrapping is plotted vs. the initial mass of the PNS, for intermediate and strong coupling, respectively. Fig. 25] shows the net energy release when PNS cool from a given entropy per baryon of 1 or 2 to zero and the neutrinos are untrapped, $Y_{L_{e}}=0.4 \rightarrow 0$.

\section{CONCLUSIONS}

We have studied the effect of finite neutrino chemical potentials on the phase diagram and the finitetemperature EoS of three-flavor quark matter with selfconsistently determined quark masses and pairing gaps. We confirm the results of Rüster et al. [25] that the phase transition to strange quark matter, such as the CFL phase is shifted to higher densities when neutrinos are trapped in the system, thus making it unlikely

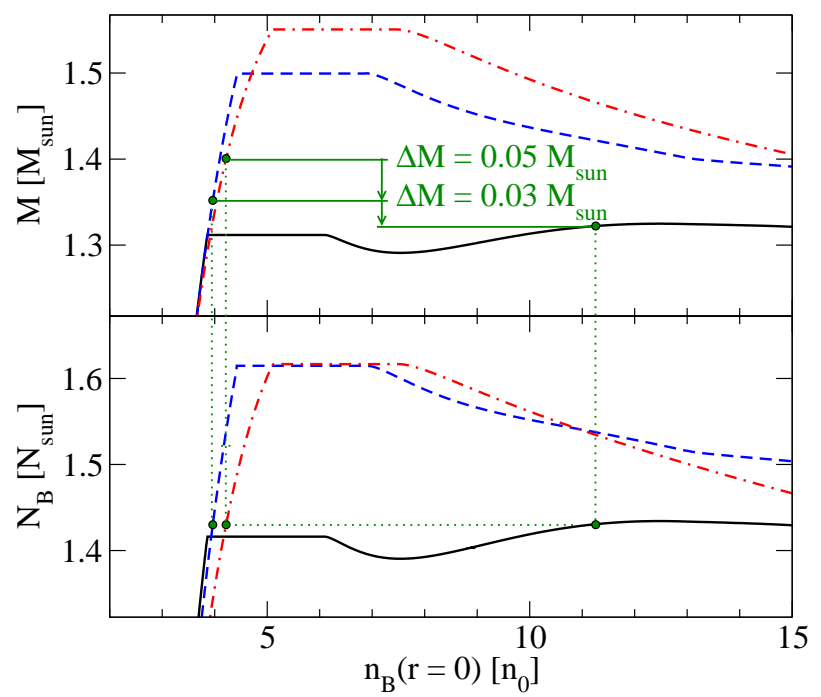

FIG. 21: (Color online) The mass defect due to cooling and neutrino untrapping at strong coupling, $\eta=1.0$. The dashdotted line represents the sequence of hot $(T=40 \mathrm{MeV})$ PNS with trapped neutrinos $\left(\mu_{\nu}=200 \mathrm{MeV}\right)$. The PNS cool rapidly by neutrino emission. A few seconds after birth the stars are cold on the nuclear scale $(T \sim \mathrm{MeV})$ and further cooling has no direct impact on their structure. However, the neutrino mean-free path is sensitive to the temperature. Below some critical temperature $(\sim \mathrm{MeV})$ the neutrinos that are trapped in the PNS escape and this has an effect on the structure. The dashed (solid) line represent the sequence of cold stars with trapped (untrapped) neutrinos, i.e., $T=0$ and $\mu_{\nu}=200 \mathrm{MeV}\left(\mu_{\nu}=0\right)$. When a hot isothermal $1.40 \mathrm{M}_{\odot} \mathrm{PNS}$ with baryon number $N=1.43 \mathrm{~N}_{\odot}$ cools to low temperature by neutrino emission, the mass decreases to $1.35 \mathrm{M}_{\odot}$. At low temperature the neutrinos escape from the star and the mass decreases to $1.32 \mathrm{M}_{\odot}$. The relative mass defects are $3.5 \%$ due to cooling, and $2.1 \%$ due to neutrino untrapping.

that strange matter exist in PNS cores. We find that in the presence of trapped neutrinos, the isospin mismatch induced by the $\beta$-equilibrium conditions is reduced and the 2SC phase becomes more favorable. In particular, the onset of the 2SC phase is shifted to lower densities in the presence of trapped neutrinos. This result seems to be robust, as it has been found also for inhomogeneous phases of the LOFF type [26]. The investigation of the neutrino trapping effect on the LOFF phases in the phase diagram with self-consistently determined strange quark masses is an interesting task for future work.

A new result of this study is the systematic analysis of the regions in the quark matter phase diagram that are realised in stable quark star cores. This analysis is carried out for two different strengths of the coupling in the diquark channel and for two different values of the neutrino chemical potential, i.e., with and without neutrino trapping. A remarkable finding is that for the initial evolution of a PNS, when the neutrinos are trapped, all configurations with a CFL core are unstable. In absence of trapped neutrinos there are small, isolated regions in the temperature-density plane where stable con- 


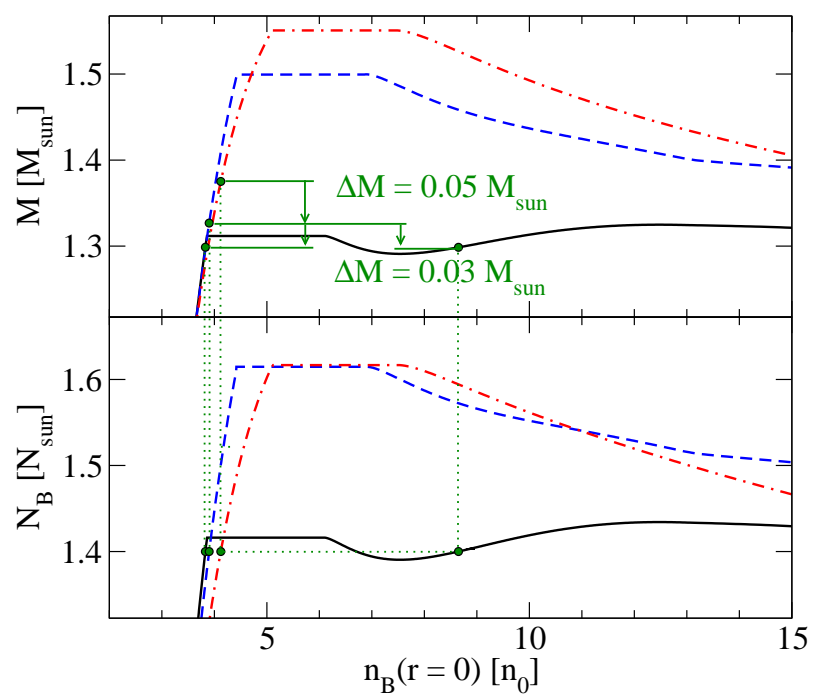

FIG. 22: (Color online) The mass defect due to cooling and neutrino untrapping at strong coupling, $\eta=1.0$. Line styles as in Fig. 21 When a hot isothermal PNS with baryon number $N=1.4 \mathrm{~N}_{\odot}$ cools to low temperature by neutrino emission, the mass decreases from $1.38 \mathrm{M}_{\odot}$ to $1.33 \mathrm{M}_{\odot}$. At low temperature the neutrinos escape from the star and the mass decreases to $1.30 \mathrm{M}_{\odot}$. The final state is either a homogenous 2SC star or a more tightly bound 2SC star with a CFL core. The mass difference between these two final states is $0.01 \%$.

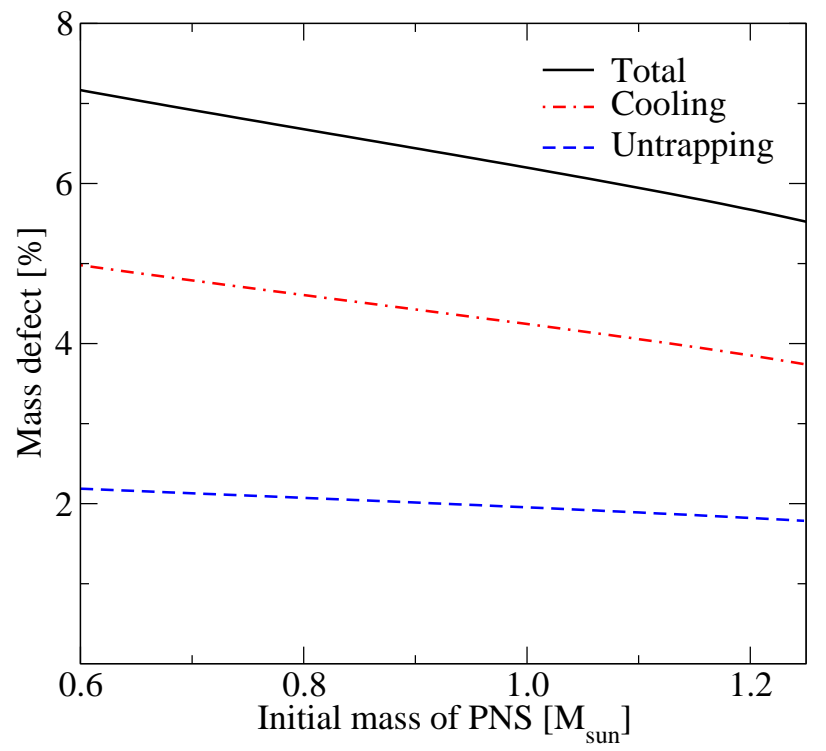

FIG. 23: (Color online) The mass defects due to cooling ( $T$ : $40 \mathrm{MeV} \rightarrow \mathrm{MeV})$ and neutrino untrapping $\left(\mu_{\nu}: 200 \mathrm{MeV} \rightarrow 0\right)$ at intermediate coupling, $\eta=0.75$.

figurations with a CFL core exists. A fraction of these 2SC-CFL stars have baryon number twins in the 2SC state. We suspect, however, that the configurations with a CFL core would be unstable when the influence of a hadronic crust is taken into account. See, e.g., Ref. 29] for an analysis of such hybrid star configurations. All

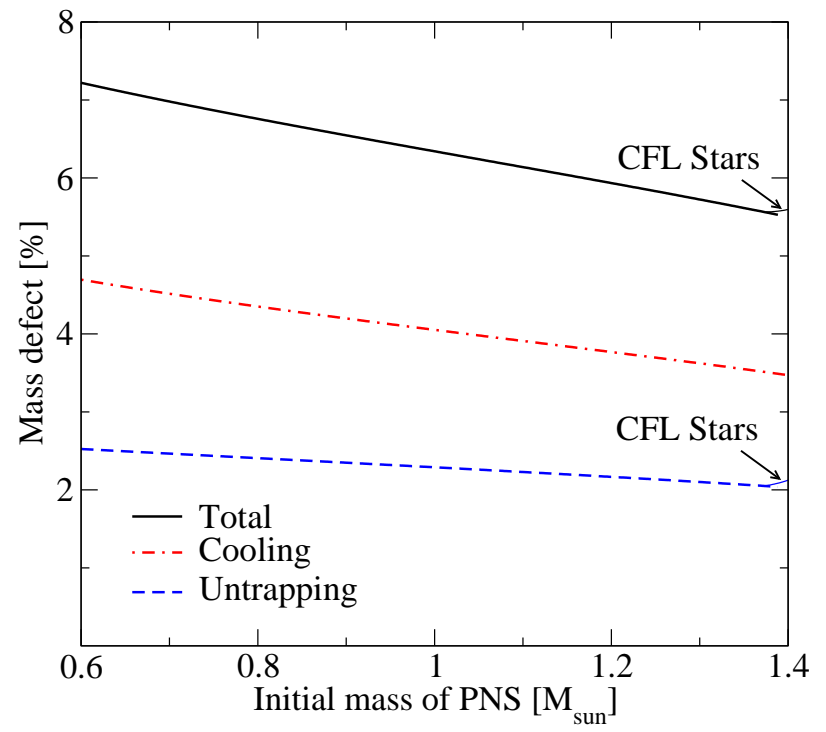

FIG. 24: (Color online) The mass defects due to cooling ( $T$ : $40 \mathrm{MeV} \rightarrow \mathrm{MeV})$ and neutrino untrapping $\left(\mu_{\nu}: 200 \mathrm{MeV} \rightarrow 0\right)$ at strong coupling, $\eta=1.0$.

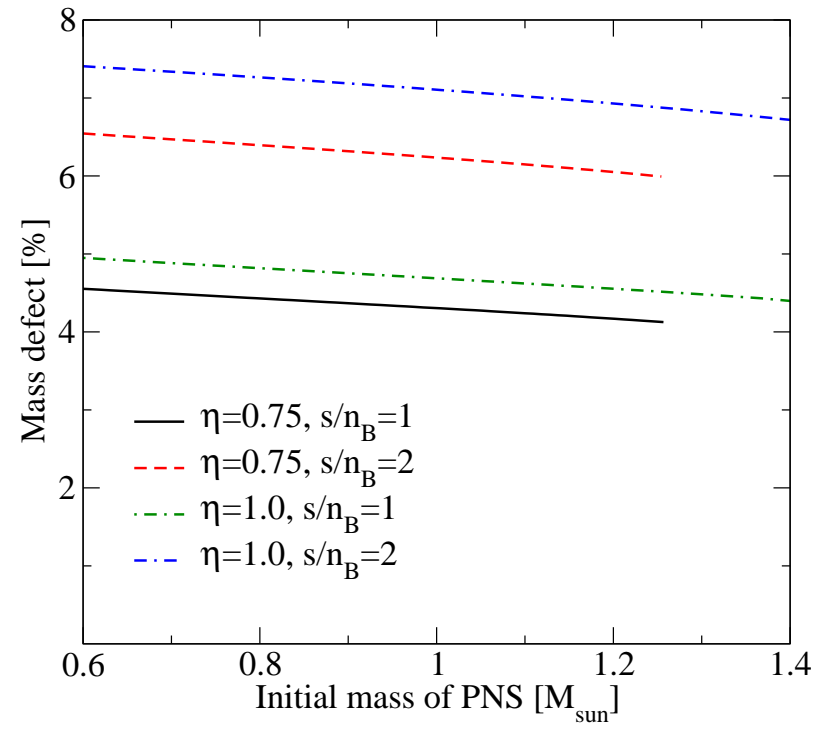

FIG. 25: (Color online) The total mass defects due to cooling $\left(s\left(T, \mu_{\nu}\right) / n_{B}\left(T, \mu_{\nu}\right): 1\right.$ or $\left.2 \rightarrow 0\right)$ and neutrino untrapping $\left(Y_{L e}\left(T, \mu_{\nu}\right): 0.4 \rightarrow 0\right)$ at intermediate, $\eta=0.75$, and strong coupling, $\eta=1.0$.

2SC quark star solutions considered in this work are stable, while the phase border in-between the $2 \mathrm{SC}$ and $\mathrm{CFL}$ phases marks the endpoint of stability for PNS sequences in the plane of central temperature and central quark number chemical potential.

The maximum temperature of PNS are estimated to about $50 \mathrm{MeV}$ for an entropy per baryon of 3, which is an upper limit for the entropy generated in the adiabatic compression of the core during collapse of a massive star. For this value of the entropy per baryon, the temperature 
in the CFL phase could reach about $65 \mathrm{MeV}$, but the corresponding PNS configurations are unstable.

When hot PNS cool by neutrino emission and reaches the neutrino opacity temperature, $T_{c} \sim 1 \mathrm{MeV}$, i.e., the temperature where the neutrino mean free path becomes comparable to the size of the quark star core, the neutrinos created by electron capture in the collapse are untrapped. By calculating and comparing the compact star sequences for different neutrino untrapping scenarios we find that the energy release due to neutrino untrapping (and/or cooling) is of the order of 100 bethe. This process could therefore be important for the inner engine of supernovæ and gamma-ray bursts.

The discussion of superconducting quark matter phases in the present work concerns the stability of PNS configurations with a CFL core and a neutrino heating mechanism for the outer core of newborn PNS due to the fact that the opacity temperature in (superconducting) quark matter exceeds that in nuclear matter. A detailed study of the neutrino untrapping transition in hybrid stars is beyond scope of the present work and will be given elsewhere.

\section{ACKNOWLEDGEMENTS}

F.S. acknowledges support from the Swedish Graduate School of Space Technology and the Royal Swedish Academy of Sciences. D.B. is grateful for support from the Polish Ministry of Science and Higher Education.
[1] H.-Th. Janka, K. Langanke, A. Marek, G. MartínezPinedo, B. Müller, arXiv:astro-ph/0612072

[2] J. M. Lattimer and M. Prakash, arXiv:astro-ph/0612440.

[3] A. Burrows and J. M. Lattimer, Astrophys. J. 307, 178 (1986).

[4] M. Prakash, I. Bombaci, M. Prakash, P. J. Ellis, J. M. Lattimer and R. Knorren, Phys. Rept. 280, 1 (1997) arXiv:nucl-th/9603042.

[5] K. Rajagopal and F. Wilczek, arXiv:hep-ph/0011333

[6] M. G. Alford, Ann. Rev. Nucl. Part. Sci. 51, 131 (2001) arXiv:hep-ph/0102047.

[7] T. Schafer, arXiv:hep-ph/0304281

[8] D. H. Rischke, Prog. Part. Nucl. Phys. 52, 197 (2004) arXiv:nucl-th/0305030.

[9] R. Casalbuoni and G. Nardulli, Rev. Mod. Phys. 76, 263 (2004) arXiv:hep-ph/0305069

[10] M. Buballa, Phys. Rept. 407, $205 \quad$ (2005) arXiv:hep-ph/0402234.

[11] M. Huang, Int. J. Mod. Phys. E 14, 675 (2005) arXiv:hep-ph/0409167.

[12] I. A. Shovkovy, Found. Phys. 35, 1309 (2005) |arXiv:nucl-th/0410091].

[13] I. Bombaci and B. Datta, Astrophys. J. 530, L69 (2000) arXiv:astro-ph/0001478.

[14] Z. Berezhiani, I. Bombaci, A. Drago, F. Frontera, and A. Lavagno, Astrophys. J. 586, 1250 (2003).

[15] D. N. Aguilera, D. Blaschke and H. Grigorian, Astron. \& Astrophys. 416, 991 (2004); arXiv:astro-ph/0212237.

[16] A. Drago, A. Lavagno and G. Pagliara, Nucl. Phys. A 774, 823 (2006) |arXiv:astro-ph/0510018.

[17] A. Drago, G. Pagliara and I. Parenti, arXiv:astro-ph/0608224

[18] J. Berdermann, D. Blaschke, H. Grigorian and D. N. Voskresensky,

Prog. Part. Nucl. Phys. 57, 334 (2006); arXiv:astro-ph/0512655.
[19] H. Abuki, M. Kitazawa and T. Kunihiro, Phys. Lett. B 615, 102 (2005) arXiv:hep-ph/0412382.

[20] S. B. Rüster, V. Werth, M. Buballa, I. A. Shovkovy and D. H. Rischke, Phys. Rev. D 72, 034004 (2005) arXiv:hep-ph/0503184.

[21] D. Blaschke, S. Fredriksson, H. Grigorian, A. M. Öztas and F. Sandin, Phys. Rev. D 72, 065020 (2005) arXiv:hep-ph/0503194.

[22] H. Abuki and T. Kunihiro, Nucl. Phys. A 768, 118 (2006) arXiv:hep-ph/0509172.

[23] H. J. Warringa, D. Boer and J. O. Andersen, Phys. Rev. D 72, 014015 (2005) arXiv:hep-ph/0504177.

[24] A. W. Steiner, S. Reddy and M. Prakash, Phys. Rev. D 66, 094007 (2002) |arXiv:hep-ph/0205201].

[25] S. B. Rüster, V. Werth, M. Buballa, I. A. Shovkovy and D. H. Rischke, Phys. Rev. D 73, 034025 (2006) arXiv:hep-ph/0509073.

[26] V. Laporta and M. Ruggieri, Phys. Lett. B 633, 734 (2006) [Erratum-ibid. B 637, 383 (2006)] arXiv:hep-ph/0511155.

[27] A. I. Larkin and Yu. N. Ovchinnikov, Zh. Eksp. Teor. Fiz. 47, 1136 (1964); P. Fulde and R. A. Ferrell, Phys. Rev. 135, A550 (1964).

[28] M. Buballa and I. A. Shovkovy, Phys. Rev. D 72, 097501 (2005) arXiv:hep-ph/0508197.

[29] T. Klähn et al., arXiv:nucl-th/0609067

[30] F. Sandin and A. M. Oztas, Phys. Rev. C 73, 035203 (2006) [arXiv:hep-ph/0512087].

[31] Note that a gapless CFL phase exist at low temperature for intermediate coupling and $\mu_{\nu}=200 \mathrm{MeV}$. This is irrelevant for the evolution of PNS for two reasons: (1) at very low $T$ neutrinos are not trapped and (2) the corresponding quark star configurations are unstable. In addition, low-temperature gapless phases suffer from a chromomagnetic instability and are typically unphysical. 